\title{
Polarization dependence of angle-resolved photoemission with submicron spatial resolution reveals emerging one-dimensionality of electrons in $\mathrm{NbSe}_{3}$
}

\author{
M. A. Valbuena, ${ }^{1,2,{ }^{*}, \dagger}$ P. Chudzinski, $,{ }^{3,4,}{ }^{*},{ }^{\dagger}$ S. Pons, ${ }^{1,5}$ S. Conejeros, ${ }^{6}$ P. Alemany,${ }^{7}$ E. Canadell,${ }^{8}$ H. Berger, ${ }^{1}$ E. Frantzeskakis,${ }^{9}$ \\ J. Avila, ${ }^{9}$ M. C. Asensio, ${ }^{9,}$ T. Giamarchi, ${ }^{10}$ and M. Grioni ${ }^{1}$ \\ ${ }^{1}$ Institute of Physics (IPHYS), Ecole Polytechnique Fédérale de Lausanne (EPFL), CH-1015 Lausanne, Switzerland \\ ${ }^{2}$ Catalan Institute of Nanoscience and Nanotechnology (ICN2), CSIC and The Barcelona Institute of Science and Technology, \\ Campus UAB, Bellaterra, 08193 Barcelona, Spain \\ ${ }^{3}$ Institute for Theoretical Physics, Center for Extreme Matter and Emergent Phenomena, Utrecht University, \\ Leuvenlaan 4, 3584 CE Utrecht, The Netherlands \\ ${ }^{4}$ School of Mathematics and Physics, Queen's University Belfast, Belfast BT7 1NN, Northern Ireland, United Kingdom \\ ${ }^{5}$ Laboratoire de Physique et d'Études des Matériaux, École de Physique et Chimie Industrielles de la ville de Paris, ESPCI, \\ PSL Research University; CNRS UMR8213; Sorbonne Universités, UPMC University, 10 rue Vauquelin, 75005 Paris, France \\ ${ }^{6}$ Departamento de Química, Universidad Católica del Norte, Av. Angamos 0610, Antofagasta 124000, Chile \\ ${ }^{7}$ Departament de Ciència de Materials i Química Física and Institut de Química Teòrica i Computacional (IQTCUB), \\ Universitat de Barcelona, Martí i Franqués 1, 08028 Barcelona, Spain \\ ${ }^{8}$ Institut de Ciència de Materials de Barcelona (ICMAB-CSIC), Campus UAB, 08193 Bellaterra (Barcelona), Spain \\ ${ }^{9}$ Synchrotron SOLEIL, L'Orme des Merisiers, Saint-Aubin-BP 48, 91192 Gif sur Yvette, France \\ ${ }^{10}$ Department of Quantum Matter Physics, University of Geneva, 24 Quai Ernest-Ansermet, 1211 Geneva, Switzerland
}

(Received 28 February 2018; published 8 February 2019)

\begin{abstract}
In materials with nearly commensurate band filling the electron liquid may spontaneously separate into components with distinct properties, yielding complex intra- and interunit cell ordering patterns and a reduced dimensionality. Polarization-dependent angle-resolved photoemission data with submicron spatial resolution demonstrate such an electronic self-organization in $\mathrm{NbSe}_{3}$, a compound considered to be a paradigm of charge order. The new data indicate the emergence of a novel order, and reveal the one-dimensional (1D) physics hidden in a material which naively could be considered the most three dimensional of all columnar chalcogenides. The 1D physics is evidenced by a new selection rule-in two polarizations we observe two strikingly different dispersions each closely resembling apparently contradicting results of previous studies of this material.
\end{abstract}

DOI: 10.1103/PhysRevB.99.075118

\section{INTRODUCTION}

Recent developments-e.g., in the field of cuprates [1-6] and pnictides $[7,8]$ — prompt us to seriously consider whether, in an ordered phase, the electrons can self-differentiate in such a way that only some of them develop an order and coexist [9-11] with the remaining nongapped carriers [12]. This scenario is of uttermost importance in materials with reduced dimensionality where interactions can particularly easily lead to new exotic instabilities [13-15], but these systems are both fragile with respect to dimensional crossover $[16,17]$, and prone to coupling to lattice vibrations. This is unfortunate, because they completely preempt all exciting

${ }^{*}$ M.A.V. and P.C. contributed equally to this work.

${ }^{\dagger}$ Present address: IMDEA Nanociencia, C/ Faraday 9, Ciudad Universitaria de Cantoblanco, 28049, Madrid, Spain.

¥Corresponding author: p.chudzinski@qub.ac.uk

§Present address: Instituto de Ciencia de Materiales de Madrid (ICMM) from the Consejo Superior de Investigaciones Científicas (CSIC), Calle Sor Juana Inés de la Cruz, 3 - Cantoblanco 28049 Madrid, Spain.
non-Fermi liquid phenomena which could take place at the lowest energies [18-20].

Materials where distinct carriers independently governed by each of these perturbations can potentially coexist, give us an opportunity to overcome this long-standing stalemate. Here we report angle-resolved photoemission spectroscopy measurements with sub- $\mu \mathrm{m}$ spatial resolution (nanoARPES) of $\mathrm{NbSe}_{3}$, a material that has been considered a paradigm of charge order. Our new data question a canonical scenario for charge-density-wave (CDW) formation [21] and suggests the emergence of a novel kind of order. The resulting electrons' self-organization reveals the one-dimensional (1D) physics hidden in a material which naively could be considered to be the most three dimensional (3D) of all columnar chalcogenides.

The quasi-1D compound $\mathrm{NbSe}_{3}$ has a columnar structure, shown in Fig. 1(a) [22]. It undergoes two successive CDW transitions, which affect electrons on distinct columns [23-25], at $T_{\mathrm{CDw} 1}=145 \mathrm{~K}$ with wave vector $\mathbf{q}_{1}=$ $(0,0.241,0)$, and at $T_{\mathrm{CDW} 2}=59 \mathrm{~K}$, with wave vector $\mathbf{q}_{2}=$ $(0.5,0.259,0.5)[26,27]$. The resulting charge ordering is not strong enough to remove the entire FS [26,28], and metallic conduction is observed even below $T_{\mathrm{CDW} 2}$. The instabilities are usually interpreted in terms of imperfect nesting of the 
(a)

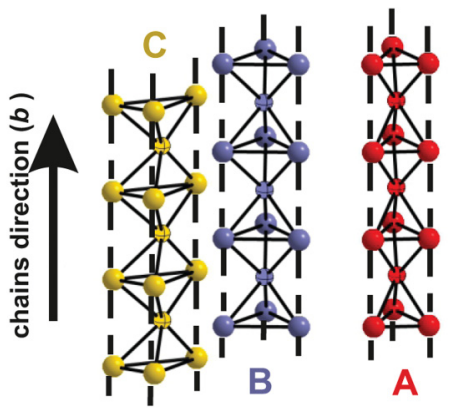

(b)

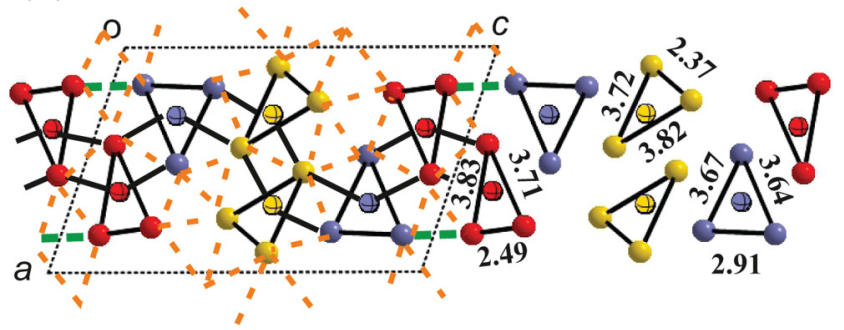

FIG. 1. (a) The crystal structure of $\mathrm{NbSe}_{3}$ (monoclinic, $a=$ $10.01 \AA, b=3.48 \AA, c=15.63 \AA, \beta=109.5^{\circ}$ ) is built out of prismatic columns of three types, hereafter labeled columns A-C, running along the $b$ axis. (b) $a c$ plane cross section. Green (orange) dashed lines indicate strong (weak) Se $\cdots$ Se intercolumnar contacts.

warped multisheet Fermi surface (FS). However, the nature of the gapless states has proven quite elusive, with several proposals for the location of residual pockets in the Brillouin zone [28-30].

Previous ARPES experiments have reported dispersive bands and CDW-related gaps [21,31] and, more recently, possible signatures of $1 \mathrm{D}$ physics [32]. In our measurements an electronic self-organization, driven by interactions, is revealed by emergent polarization-dependent selection rules, which reflect the underlying $1 \mathrm{D}$ nature of the electronic states. The number of observed bands is also doubled with respect to a single-particle picture. The ability to zoom in on a small selected, disorder-free area of the sample was crucial to observe these features, which escaped previous investigations.

The outline of the paper is as follows. After a description of the experimental methods in Sec. II we present the experimental ARPES results in Sec. III. We illustrate our DFT $+U$ study of the material in Sec. IV and derive in Sec. V the ARPES selection rules that explain the striking polarization dependence of the ARPES data. Finally, in Sec. VI we present a consistent interpretation of our both experimental and theoretical results.

\section{EXPERIMENTAL METHODS}

$\mathrm{NbSe}_{3}$ single crystals with typical dimensions of $0.01 \times$ $10 \times 0.05 \mathrm{~mm}^{3}$ grown using the chemical vapor transport method [33] were mounted on the sample holder, as shown in Fig. 2. Samples were cleaved in situ in an ultrahigh vacuum (UHV) system at room temperature to expose the $b c$ plane. As detailed below, with the help of the spatial photoemission scanning system it was possible to focus the beam on one of the best cleaved $\mathrm{NbSe}_{3}$ whiskers.

We performed nanoARPES measurements with the $k$ microscope of the ANTARES beamline at the SOLEIL syn-
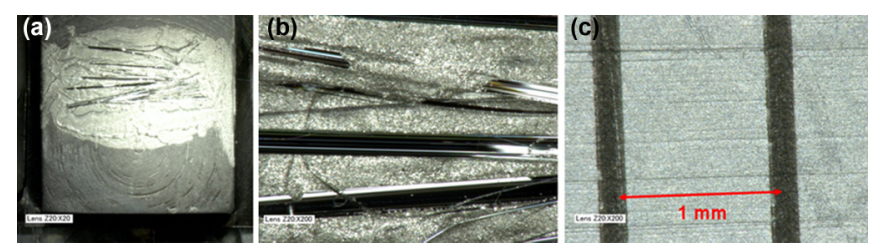

FIG. 2. (a) Sample holder with several $\mathrm{NbSe}_{3}$ samples. (b) Optical microscope image of $\mathrm{NbSe}_{3}$ whiskers. The scale of the image is given in (c).

chrotron [34,35], with spatial and energy resolution of $100 \mathrm{~nm}$ and, respectively, $20 \mathrm{meV}$. The microscopy capabilities of the experimental end station are schematically depicted in Fig. 3. A photon beam of variable energy and polarization is focused on a sub- $\mu \mathrm{m}$ spot by means of a Fresnel zone plate (ZP). An order sorting aperture (OSA) removes undesirable higher-order diffraction effects. The sample is mounted on a high-precision scanning stage with five degrees of freedom comprising linear and angular motion. Piezoscanners and an interferometric control of the sample and ZP positions guarantee zero backlash and enable sub- $\mu \mathrm{m}$ accuracy and reproducibility as different parts of the sample are scanned through the beam. All these parts ensure spatial resolution of the acquired data without sacrificing any of the qualities of conventional ARPES. Images of the sample chemical composition and angle-resolved valence band states at a sub- $\mu \mathrm{m}$ scale may be acquired by the synchronized scanning of the sample with respect to the focused beam, while the electron analyzer collects photoelectrons of a selected kinetic energy.

Spatially resolved Se $3 d$ intensity scans were obtained at different lateral scales and shown in Figs. 4(a) and 4(b). The integrated intensity of the Se $3 d$ core level spectrum is represented as a function of position in a color scale, yielding a "real-space" mapping of $\mathrm{NbSe}_{3}$ micrometric fibers. Figure 4(c) shows the Se $3 d$ core level spectrum acquired in a high-intensity area in one of the best quality chains (red area). The solid line is the result of a fit of the spectrum with five spin-orbit doublets. The complex line shape, which reflects inequivalent Se sites, is a direct indication of the quality of the photoemission data acquired with sub- $\mu \mathrm{m}$ lateral resolution.

\section{ARPES RESULTS: POLARIZATION DEPENDENT BAND DISPERSION}

ARPES measurements of $\mathrm{NbSe}_{3}$ are challenging. Crystals grow in the form of very thin whiskers, parallel to the $b$ axis, that assemble in bundles. It is thus difficult to ensure that standard ARPES results are not affected by inhomogeneities or by averaging over several domain orientations. In our experiment we took advantage of the sub- $\mu \mathrm{m}$ photon spot size to select a flat homogeneous area of a well-cleaved single crystal whisker. This is especially important for the analysis of the dependence of the spectra on the light polarization, which will be presented below. Data were collected at $100 \mathrm{eV}$ photon energy with light polarized either within ( $p$ polarization) or perpendicular to ( $s$ polarization) the horizontal scattering plane, which contained the $b$ axis of the sample.

Figure 5 shows ARPES intensity maps measured at $55 \mathrm{~K}$, below $T_{\mathrm{CDW} 2}$, along the $b^{*} \| b$ axis. We used a soft median 


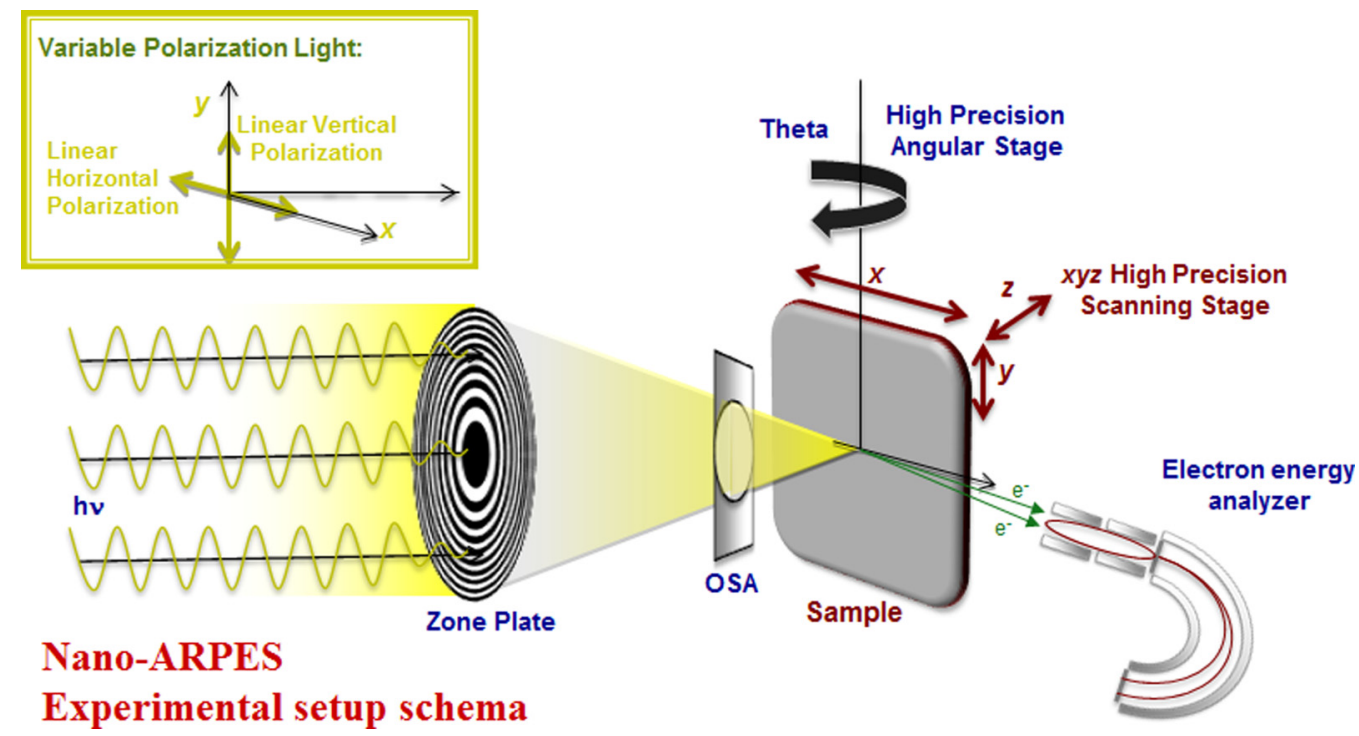

FIG. 3. Sub- $\mu \mathrm{m}$ ARPES experimental setup scheme. The incoming light is focused in a sub- $\mu \mathrm{m}$ region by a zone plate. The sample is mounted on a high-precision 5 degrees of freedom manipulator. Linear horizontal and linear vertical polarized light is available.

filter to remove spikes, and then symmetrized the data around $k=0$. The average incoherent background, estimated from the noise level at $k>0.6 \AA$, is monotonous and can be fitted with a Shirley function, which was then subtracted from the ARPES intensity. Curvature plots [36] extracted from Figs. 5(a) and 5(b) to enhance the visibility of the bands are shown in Figs. 5(c) and 5(e) in a smaller region around $\Gamma$. Figures $6(\mathrm{a})$ and $6(\mathrm{~b})$ show the corresponding raw, nonsymmetrized, data, and Figs. 6(c) and 6(d) the energy distribution curves (EDCs) extracted from those maps. For a quantitative analysis we fitted the line shapes of EDCs and momentum distribution curves (MDCs) extracted from
ARPES intensity maps to determine the energy and wave vector of the dispersive features. The resulting peak positions are shown with different symbols in Figs. 5(d) and 5(f) and by colored tick marks on the EDCs Figs. 6(c) and 6(d).

For $s$ polarization, in Figs. 5(c) and 5(d), we observe three parabolic bands, labeled $\mathrm{A} 1^{+}, \mathrm{C} 2$, and $\mathrm{A} 3^{+}$, near the Fermi energy $\left(E_{F}\right)$. Extrapolating their dispersions to $E_{F}$ yields $k_{F 1}=0.14 \pi / b, k_{F 2}=0.22 \pi / b$, and $k_{F 3}=0.37 \pi / b$. A gap opens at $E_{F}$ in band $\mathrm{C} 2$ owing to the change of periodicity induced by CDW1. Further analysis of the data at $k_{F 1}$ and at $k_{F 3}$ reveals a reduction of spectral weight near $E_{F}$ (see, e.g., Figs. 9 and 10). This suggests the incipient opening of (a) $\quad \mathrm{NbSe}_{3}$

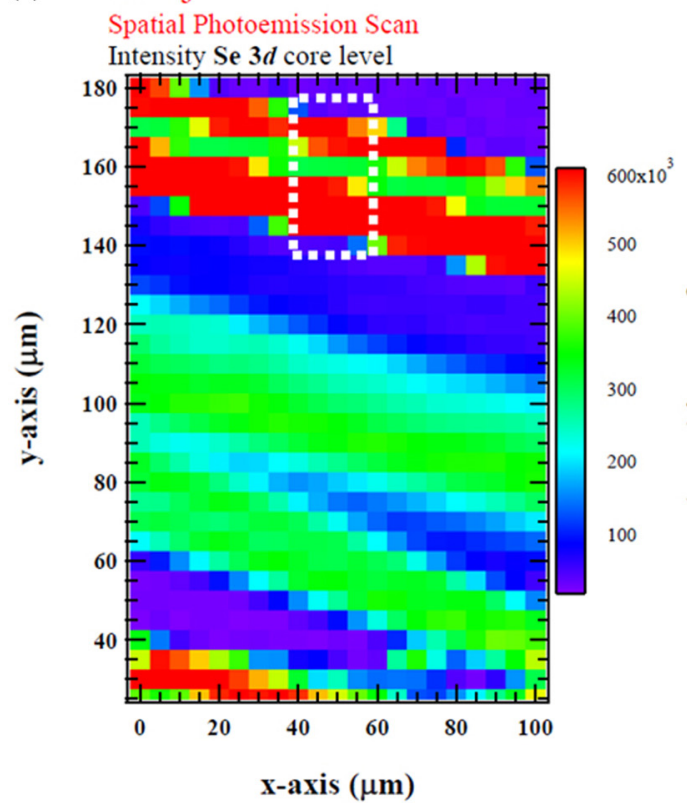

(b)

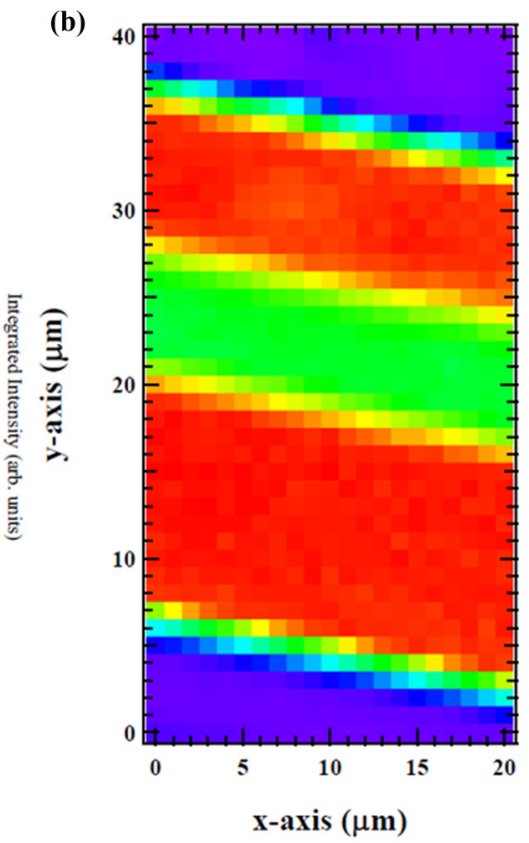

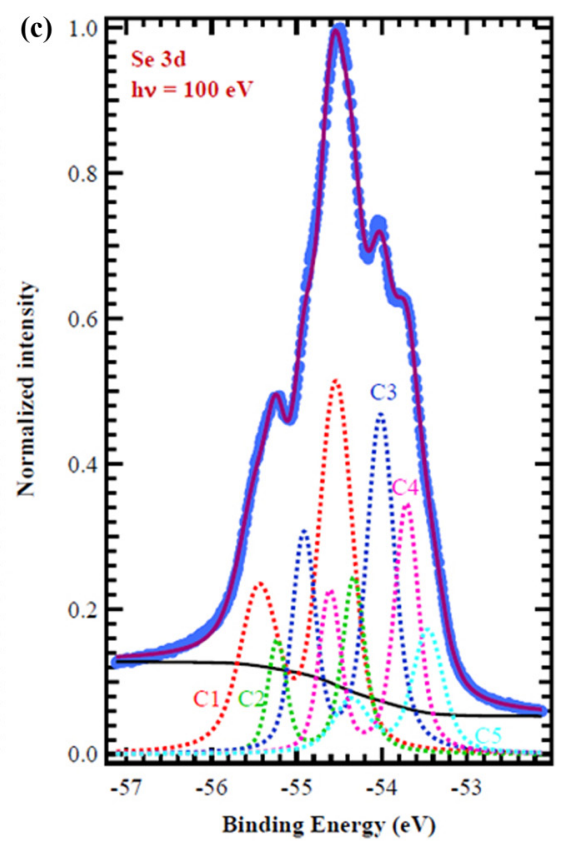

FIG. 4. (a) Spatially resolved Se $3 d$ intensity scan on $\mathrm{NbSe}_{3}$ whiskers, and (b) higher resolution scan of dashed area. (c) Se $3 d$ core level and the result of a fit with five spin-orbit doublets. 

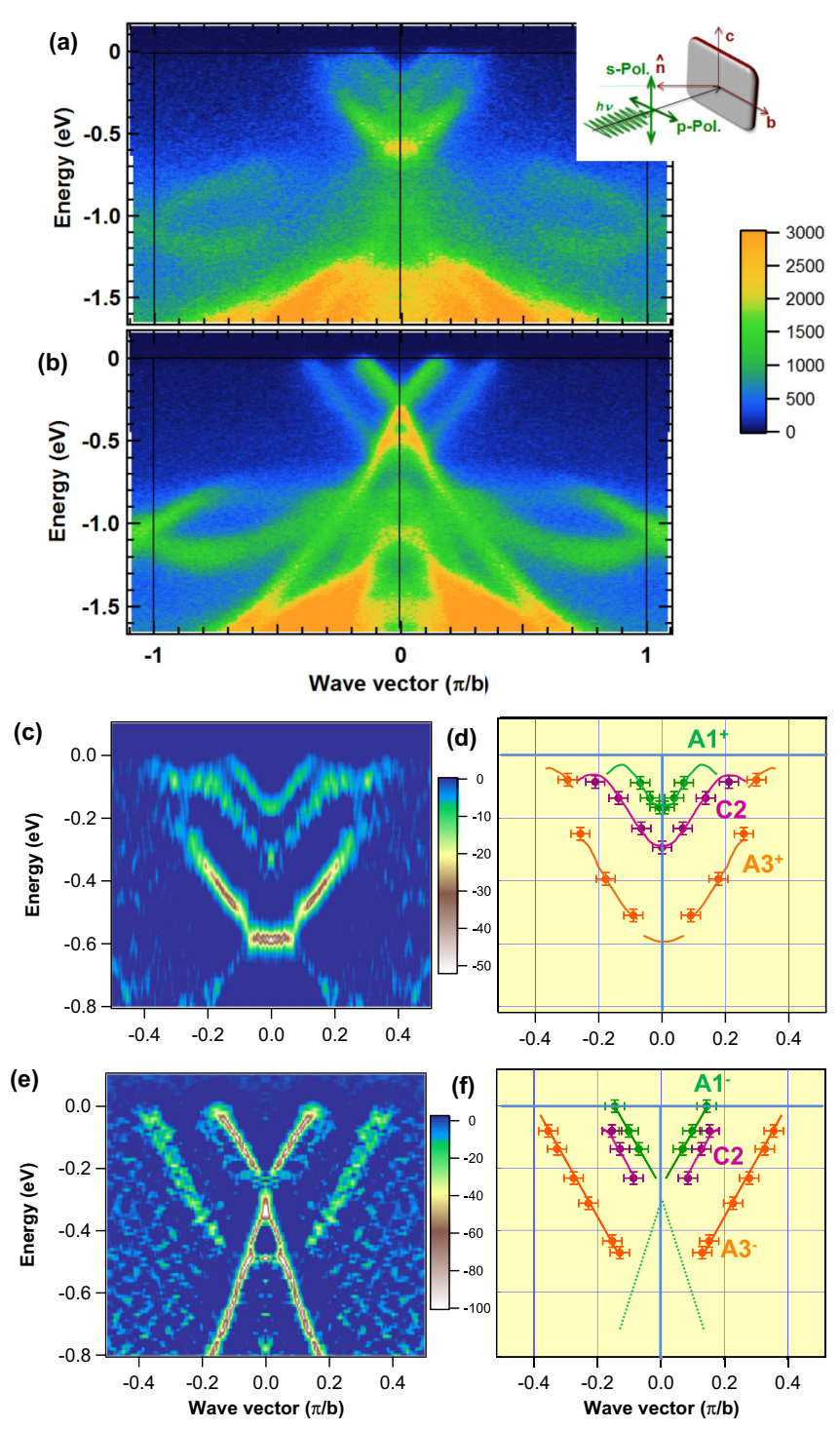

FIG. 5. ARPES intensity maps $(h v=100 \mathrm{eV} ; T=55 \mathrm{~K})$ measured along the $b^{*} \| b$ axis $(\pi / b=0.89 \AA$ ) with (a) $s$ polarization and (b) $p$ polarization. The inset shows the experimental geometry. The $s$ polarization is perpendicular to the long axis (i.e., to the $b$ axis) of the $\mathrm{NbSe}_{3}$ crystalline whiskers. The $p$ polarization has components along the $b$ axis and perpendicular to the $b c$ cleavage plane. (c) and (e) Curvature plots obtained from (a) and (b), respectively. Peak positions determined from EDCs and MDCs are shown in (d) and (f). The lines are guides to the eye.

gaps due to CDW2, which were estimated at $\approx 20 \mathrm{meV}$ by low-temperature ARPES measurements [21,31].

Data taken with $p$ polarization, in Figs. 5(b), 5(e) and 5(f) and Figs. 6(b) and 6(d), present a quite different picture. We now observe two linearly dispersing bands, labeled $\mathrm{A}^{-}$and $\mathrm{A} 3^{-}$, which do not correspond to any of the spectral features observed with $s$ polarization for the same area of the single crystal, and cross $E_{F}$ with no indications of CDW-related gaps. This dramatic polarization dependence suggests that two different types of carriers, separable by some selection rule, are simultaneously present in a homogenous crystal.
The accurate determination of the Fermi energy is crucial to address the behavior of the electronic states affected by CDW instabilities in low dimensional materials. The Fermi level position in Figs. 6(c) and 6(d) was determined from the Fermi cutoff of a polycrystalline gold sample. We have verified that it coincides with an internal energy reference extracted from the raw data. To this end we have quantitatively analyzed the energy-momentum dispersion of the linear band $\mathrm{A} 1^{-}$, which provides a clear and distinct ARPES signal. We have extracted from the intensity map of Fig. 6(b) MDCs at various energies for the $k>0$ branch and integrated the intensity under the corresponding peak. The resulting energydependent momentum-integrated intensity (Fig. 7) exhibits a clear cutoff which coincides with the Fermi energy determined from the gold sample. This MDC-based $E_{F}$ determination, besides being intrinsic, is largely unaffected by possible pitfalls affecting the more standard EDC-based procedure (see, e.g., Ref. [37]).

Figure 8 presents combined images of curvature plots obtained from raw data recorded with $s$ and $p$ polarizations. The images support the idea of distinct dispersions for the bands close to the $E_{F}$ measured with the two polarizations. This is clear in particular for the $\mathrm{A} 1^{ \pm}$bands. We also notice that $\mathrm{A} 3^{+}$exhibits a large gap at $k \sim \pm 0.3 \pi / b$ associated with the CDW1 superlattice potential. By contrast, A3 ${ }^{-}$exhibits a continuous, unaffected dispersion.

Figure 9 shows the second derivative of the spectral intensity with respect to the energy near $E_{F}$. This procedure enhances the visibility of the band edges and therefore of the opening of energy gaps, same was used in Refs. [31,32]. In Fig. 9(a) we show extracted values of indication of a CDW1 gap (yellow arrow) and of CDW2 gaps (red arrows), where the latter one is on the edge of our resolution. Leaving the exact value of the CDW2 gap aside, it is well known that bands' dispersion backfolding is an equally good hallmark of the presence of CDW. With $s$ polarization the wriggling of the spectral intensity underlines the hybridization of the $\mathrm{A} 1^{+}-\mathrm{C} 2$ and $\mathrm{C} 2-\mathrm{A} 3^{+}$bands and reveals the backfolding produced by their anticrossing. By contrast, the $\mathrm{A} 1^{-}$and $\mathrm{A} 3^{-}$bands measured with $p$ polarization exhibit linear dispersion and metallic behavior. Undoubtedly, the two [Figs. 9(a) and 9(b)] represent different behavior.

For a detailed assessment of bands dispersion near $E_{F}$ it is convenient to adopt a symmetrization procedure that removes the perturbing effect of the Fermi-Dirac distribution function. Specifically, to an EDC measured at $k_{F}$ is added a mirror image of the spectrum with respect to $E_{F}$. This yields the underlying spectral function $A\left(k_{F}, E\right)$ free from the Fermi-Dirac cutoff, with the usual mild assumption $A\left(k_{F}, E\right)=A\left(k_{F},-E\right)$ The results of this procedure applied to band $\mathrm{A} 1^{ \pm}$at $k_{F 1}$ and to band $\mathrm{A} 3^{ \pm}$at $k_{F 2}$ are illustrated in Fig. 10. Symmetrization of the spectra measured with $p$ polarization yields in both cases prominent peaks centered at $E_{F}$, consistent with gapless metallic behavior. The situation is qualitatively different for the spectra measured with $s$ polarization where, even after symmetrization, one observes a nearly constant intensity at $k_{F 3}$, and even a dip at $k_{F 1}$. There is no direct evidence of CDW2 gaps, which have been estimated at $\approx 20 \mathrm{meV}$ from low-temperature ARPES data (following the same procedure as Ref. [31]). However, one must consider that our data have 

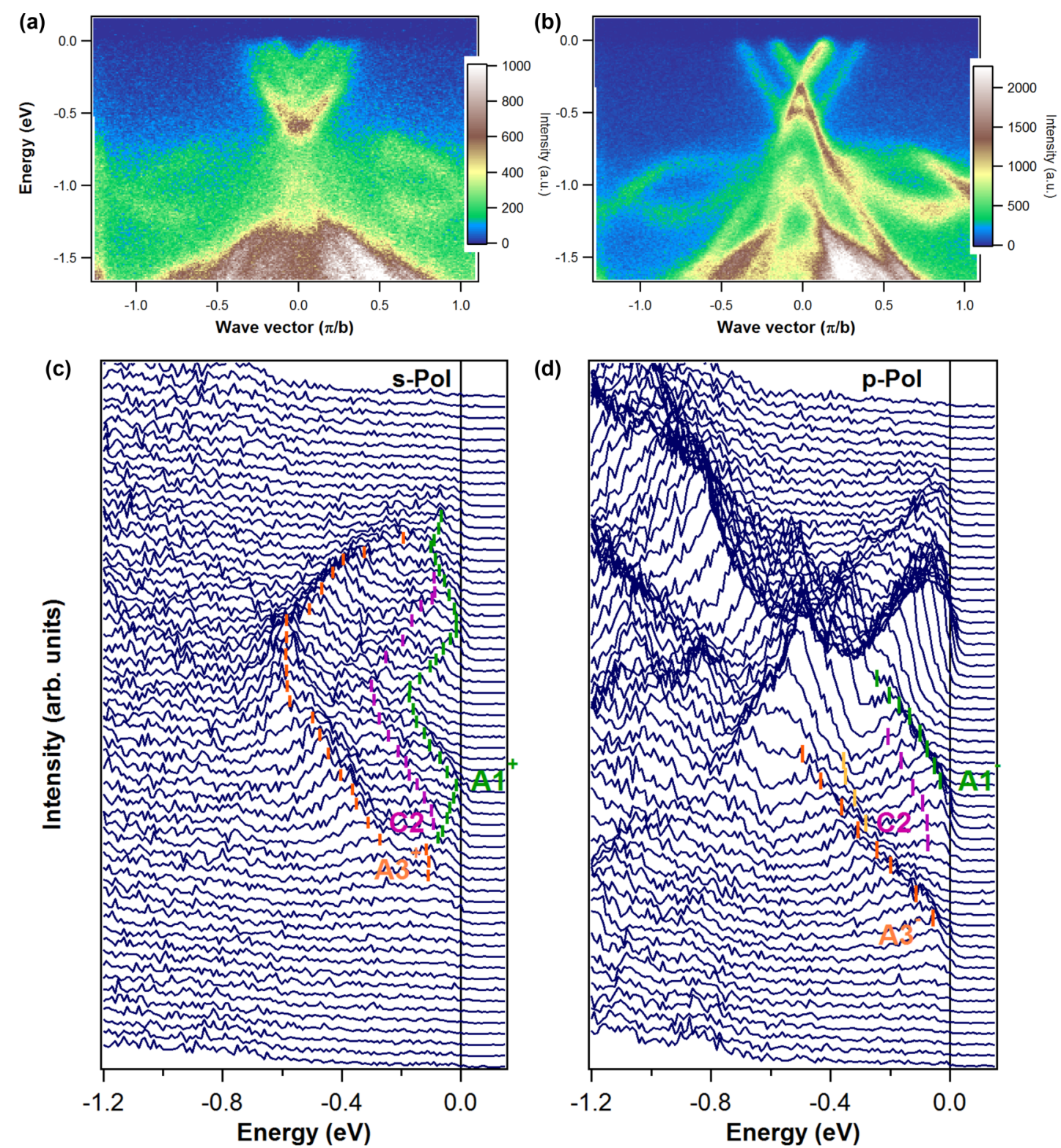

FIG. 6. ARPES intensity maps (raw data; $T=55 \mathrm{~K} ; h v=100 \mathrm{eV}$ ) for (a) $s$ polarization and (b) $p$ polarization. The collection time was $8 \mathrm{~h}$ for each map. (c) and (d) Energy distribution curves (EDCs) extracted from (a) and (b), respectively. Tick marks indicate peak positions of the various spectral features.

been measured close to $T_{\mathrm{CDW} 2}$, where the gap is smaller than its zero-temperature limit [38]. It would not be possible to observe a direct signature of such a reduced gap opening with our experimental resolution. Nevertheless, the symmetrized spectra of Fig. 10 suggest an underlying incipient gap, consistent with results shown in Fig. 9 and previous tunneling transport [38], optical spectroscopy [28,39], and spectral [31,32] properties of $\mathrm{NbSe}_{3}$. Theoretical prediction for the spectral function (without finite resolution broadening and in-gap states included) have been proposed in Ref. [40] where (in Fig. 2 therein) we observe that even very moderate temperature produces a broad EDC with rather soft gap.

The differences illustrated in Fig. 10 support the claim that the spectral features observed with different polarizations can be attributed to different bands with distinct near- $E_{F}$ properties. This conclusion is strengthened by differences in the MDCs line shapes for the two polarizations. The MDCs of Fig. 11 were extracted for $k>030 \mathrm{meV}$ below $E_{F}$, i.e., close to $E_{F}$, and yet sufficiently far to prevent artifacts due to the finite temperature and experimental resolution. Both line shapes exhibit peaks corresponding to bands $\mathrm{A} 1^{ \pm}$and $\mathrm{A} 3^{ \pm}$. Band C2 is visible as a distinct feature with $s$-polarized light but, because of matrix elements, only as a shoulder to $\mathrm{A} 1^{-}$for $p$ polarization. A comparison of the two MDCs demonstrates that the momenta of the A1 and A3 ARPES features are not the same in the two polarizations, and that therefore they must be attributed to different bands.

A further analysis of the $p$-polarization ARPES data reveals an even more complex picture. Figure 12 presents fits of MDCs extracted at selected binding energies. Best fits are obtained when two peaks, with $k$-dependent separation, are used to fit the $\mathrm{A} 3^{-}$band. They are labeled $\mathrm{A} 3_{h}^{-}$(dark orange) 


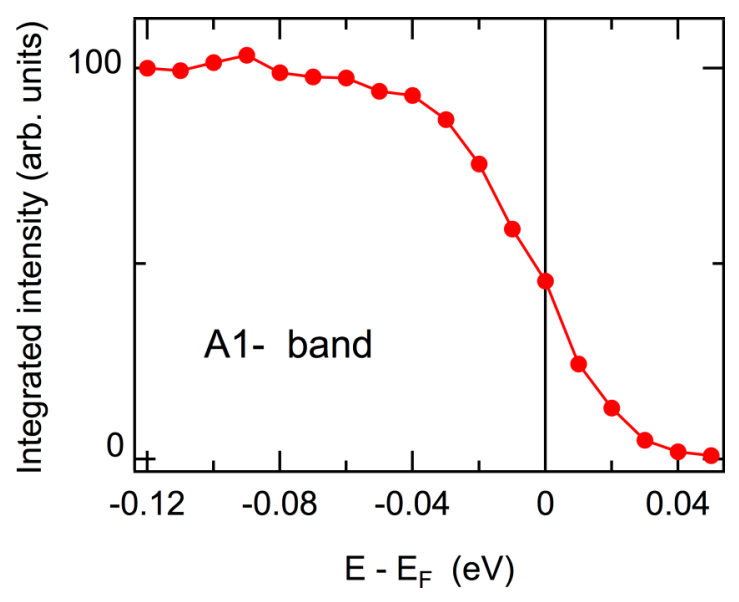

FIG. 7. Energy-dependent ARPES intensity in the $k>0$ branch of the $\mathrm{A} 1^{-}$band, obtained by integrating the corresponding peak in momentum-distribution curves (MDCs) extracted from Fig. 6(b). The inflection point determines the Fermi level position.

and $\mathrm{A} 3_{s}^{-}$(light orange). A possible interpretation can be made in terms of 1D signatures of spin-charge separation and will be discussed elsewhere [41]. The MDC fits confirm the presence of the $\mathrm{C} 2$ band, visible as a satellite of the more intense $\mathrm{A}^{-}$ band.

\section{DFT STUDY}

As a first step to interpret our data we performed $(\mathrm{GGA}+U)$ DFT calculations. Compared to previous DFT calculations on this material [21], the inclusion of a local interaction term $U$ allows us to better differentiate $\mathrm{Nb}$ atoms with formally different oxidation states. The main results are shown in Fig. 13. The present calculations were carried out using a numerical atomic orbitals density functional theory (DFT) approach $[42,43]$, which was developed for efficient calculations in large systems and implemented in the SIESTA code $[44,45]$. We have used the generalized gradient approximation (GGA) to DFT and, in particular, the functional of Perdew, Burke, and Ernzerhof [46]. Only the valence electrons are considered in the calculation, with the core being replaced by norm-conserving scalar relativistic pseudopotentials [47] factorized in the Kleinman-Bylander form [48]. We have used
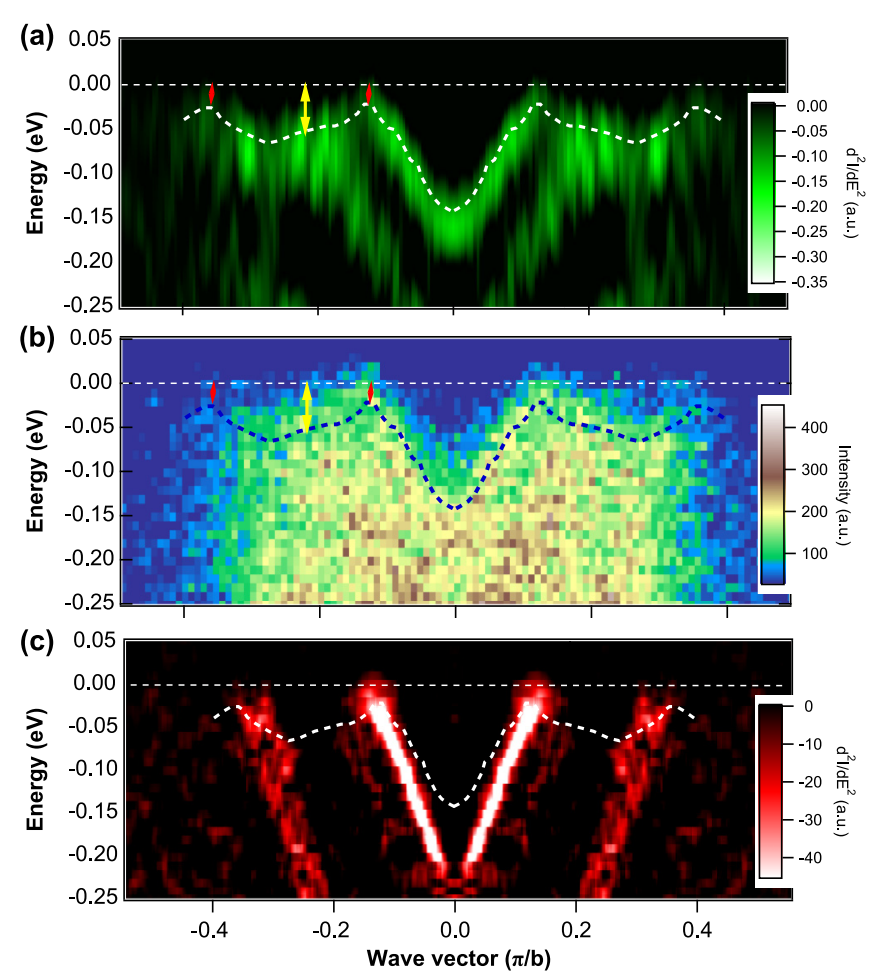

FIG. 9. (a) Second derivative (with respect to energy) of the ARPES intensity measured with $s$ polarization. (b) Corresponding raw data measured in $s$ polarization. Both (a) and (b) evidence the backfolding resulting from the hybridization of bands $\mathrm{A} 1^{+}, \mathrm{A} 2^{+}$, and $\mathrm{C} 2$. The gap amplitudes (arrows) can be estimated from the distance between the $E_{F}$ and the edge profile. (c) Second derivative with respect to energy of the ARPES intensity measured with $p$ polarization. The spectral intensity exhibits a gapless behavior and a linear dispersion with no backfolding evidences. The dashed wriggling line shown on each panel follows the inflection point of the spectral intensity at the vicinity of $E_{F}$ in the $s$ polarization.

a split-valence triple- $\zeta$ basis set including polarization orbitals with an energy shift of $50 \mathrm{meV}$ for all atoms [49]. The energy cutoff of the real space integration mesh was $250 \mathrm{Ry}$. An on-site Coulomb energy $(U)$ of $4 \mathrm{eV}$ has been taken into account within the rotationally invariant DFT $+U$ approach of Dudarev et al. [50]. The Brillouin zone was sampled using grids of $(8 \times 8 \times 8)$ and $(30 \times 30 \times 30) k$ points [51] for
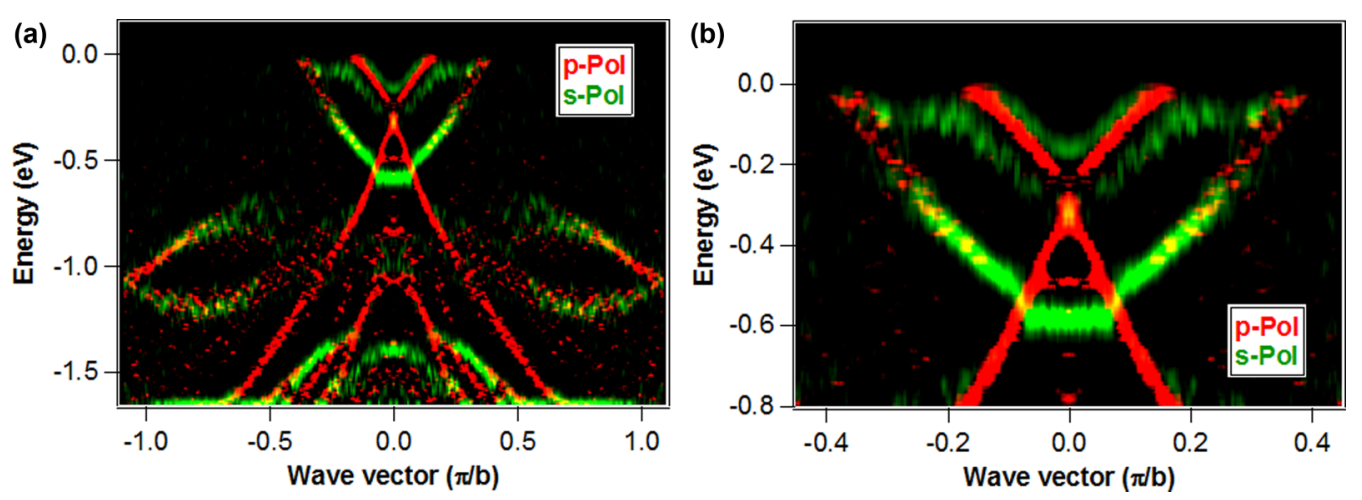

FIG. 8. (a) Comparison of curvature plots obtained from data measured with $s$ polarization (green) and $p$ polarization (red). (b) A closeup of (a) near the Fermi level. 

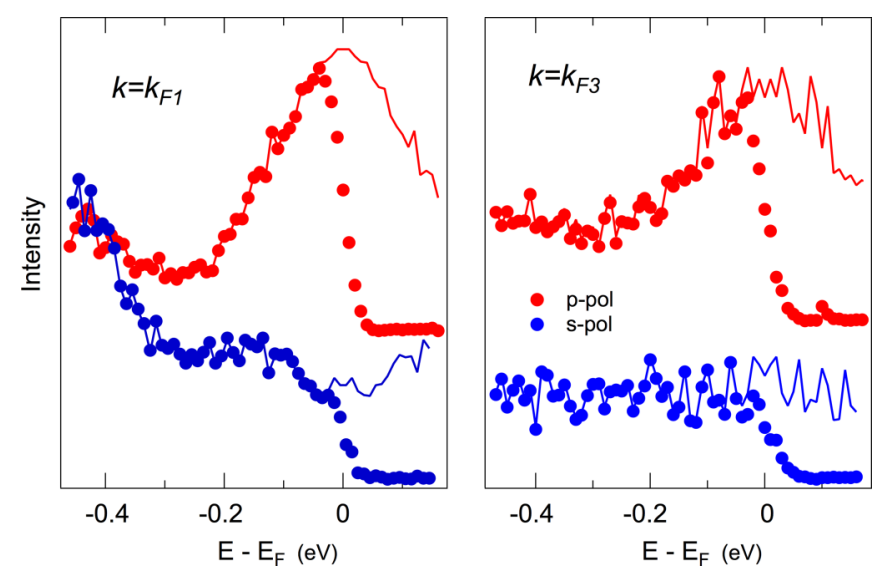

FIG. 10. (Left) EDCs measured at $k_{F 1}$ with $s$ (blue symbols) and $p$ polarization (red symbols), extracted from the intensity maps of Fig. 6. (Right) Same for $k_{F 3}$. The solid lines are obtained by symmetrizing the raw spectra around $E_{F}$.

determination of the density matrix and the Fermi surface, respectively. The x-ray crystal structure at $298 \mathrm{~K}$ was used in the calculations [22].

The calculated band structure at $298 \mathrm{~K}$ as well as its fat band analysis are reported in Figs. 13 and 14, respectively. The calculated Fermi surface as well as several particular sections are shown in Fig. 15. The band structure confirms that the $\mathrm{A}^{+}, \mathrm{A}^{+}$, and $\mathrm{C} 2$ bands observed with $s$ polarization are associated with crystal columns $\mathrm{A}$ and $\mathrm{C}$. The calculated $k_{F}$ values are in good agreement with the measured data. The two bands labeled $\mathrm{C} 2$ are degenerate near $E_{F}$ as seen in the ARPES experiment where they appear as a single band, $\mathrm{C} 2$, implying a very small hybridization within a pair of $\mathrm{C}$ columns. The bands crossing $E_{F}$ mainly derive from $d$ orbitals of a given $\mathrm{Nb}$ atom, but the $d$ weight is never more than $50 \%$, a rather different situation from, e.g., transition metal oxides. This is a manifestation of the covalent character of the $\mathrm{Nb}$-Se bonds and implies that the electron clouds are not locked on the $\mathrm{Nb} d$ orbitals, and can be deformed by the CDW potential. The band dispersion has quasi-1D character, but the anisotropy is moderate: the calculated bandwidth is $W_{b}=2.5 \mathrm{eV}$ along the $b$ axis and up to $250 \mathrm{meV}$ along

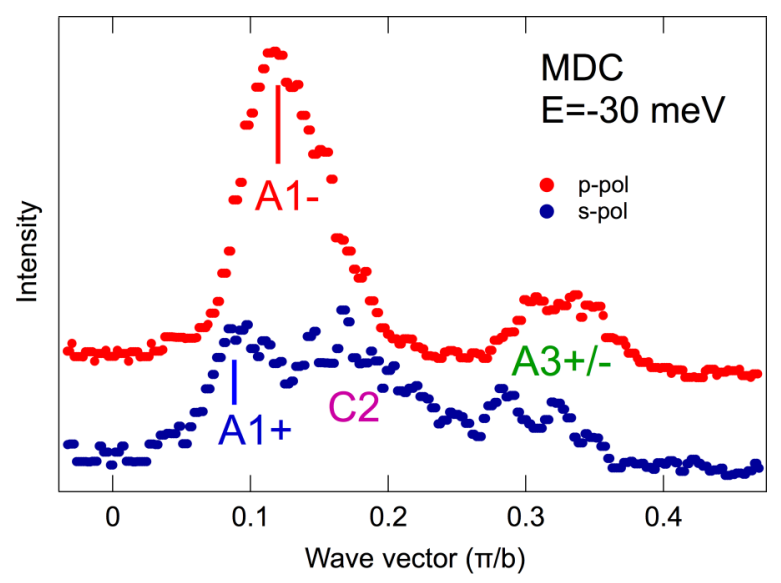

FIG. 11. MDCs extracted $30 \mathrm{meV}$ below $E_{F}$ for the two polarizations.
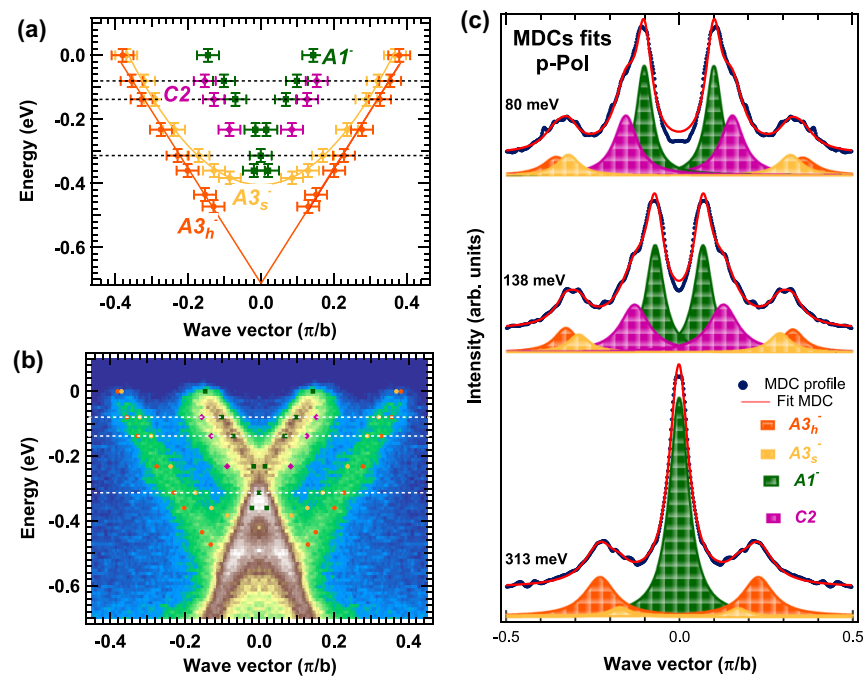

FIG. 12. (a) Band dispersion obtained from MDCs fits for $p$ polarization. (b) Results of the MDCs fit superimposed on the ARPES intensity image. The dashed lines indicates the energy profiles for MDCs shown in (c). Dark and light orange features display the double structure composing band $\mathrm{A}^{-}{ }^{-}$related to possible spinon and holon contributions.

perpendicular directions. Previously proposed band structures $[21,29,30]$ hold also when local correlations are included, but $U$ shifts band $B$ above $E_{F}$. This is consistent with scanning tunneling microscopy measurements [25] where a significant spectral weight appears just above $E_{F}$, and also with our experiment, where no extra band is detected close to the $\Gamma$ point. By contrast, the sample of Ref. [32] appears to be slightly electron doped, possibly owing to a small off-stoichiometry.

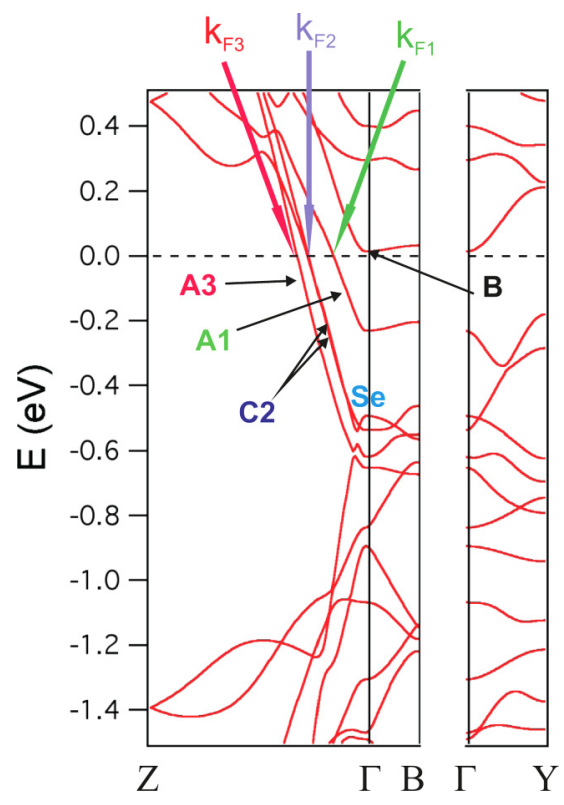

FIG. 13. Theoretical DFT GGA band calculations including finite $U=4 \mathrm{eV}$. The bands can be associated with their experimental counterparts in Fig. 5 by comparing their respective Fermi wave vectors. The energy zero corresponds to the Fermi level. $\Gamma=$ $(0,0,0), Y=(1 / 2,0,0), Z=(0,1 / 2,0), B=(0,0,1 / 2)$, and $D=$ $(0,1 / 2,1 / 2)$ in units of the monoclinic reciprocal lattice vectors. 


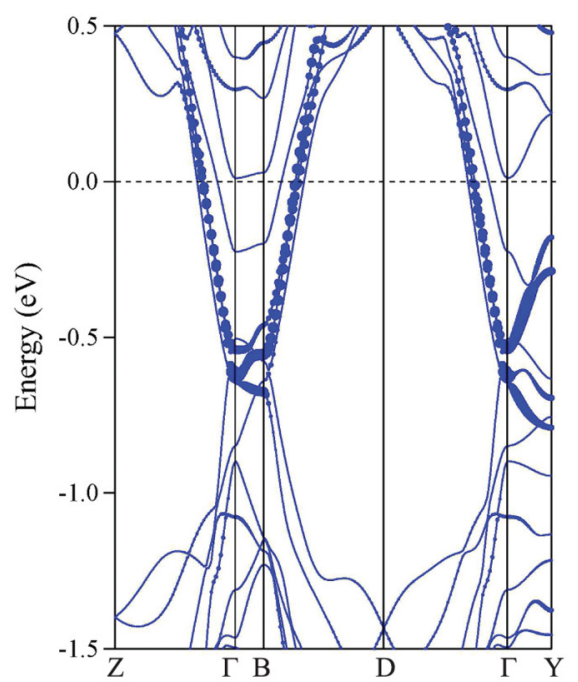

(a)

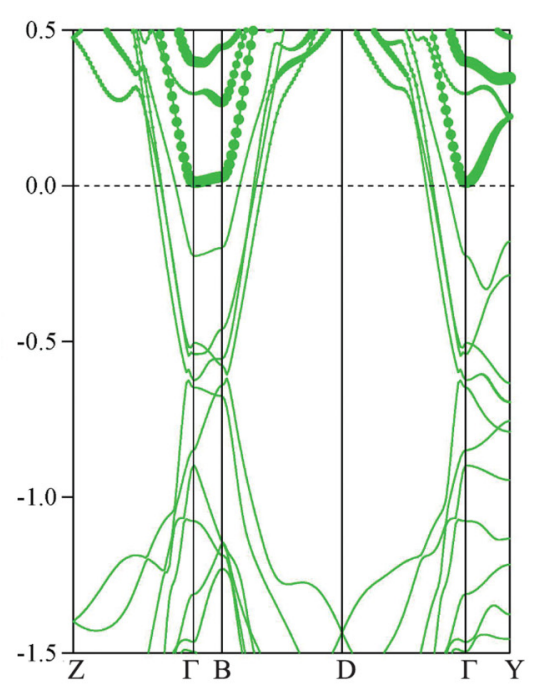

(b)

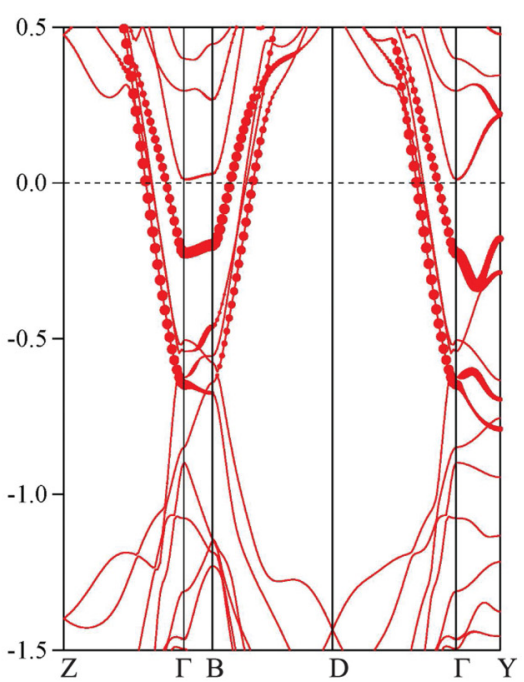

(c)

FIG. 14. Band structure for $\mathrm{NbSe}_{3}$ at $298 \mathrm{~K}$ where the size of the blue (a), green (b), and red (c) circles is proportional to the contribution of the niobium atoms in chains $\mathrm{C}, \mathrm{B}$, and $\mathrm{A}$, respectively.

\section{EMERGENT SYMMETRY RULES}

From the DFT results one could infer that, at temperatures smaller than the band dispersion, electrons should move coherently in the crystal in all directions, and in particular perpendicular to the chains. Then the symmetry of the electronic system would be that of the $P 2_{1} / m$ monoclinic space group of $\mathrm{NbSe}_{3}$, with only two mirror symmetry planes, both parallel to the $a c$ plane. Since the scattering plane in our experiment is perpendicular to these symmetry planes, no definite polarization selection rule [52] should apply and one should thus observe identical dispersions for both polarizations [53], albeit with very different intensity. This is in stark contrast with the results of Fig. 5. In this section we introduce the concept of selection rules in ARPES (Sec. V A), then

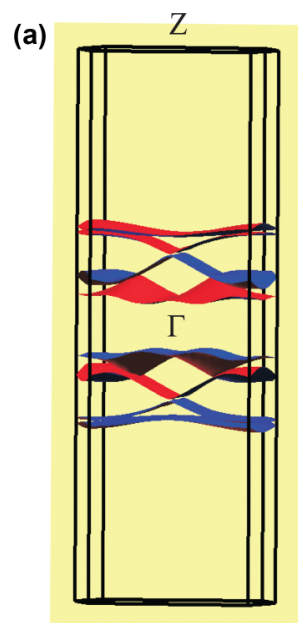

(b)
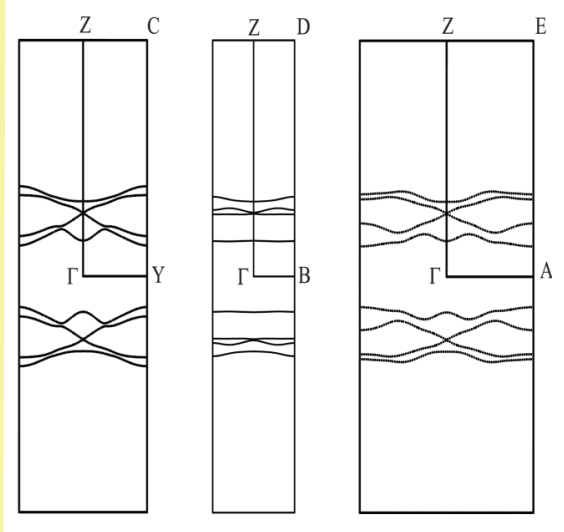

FIG. 15. (a) Calculated Fermi surface for $\mathrm{NbSe}_{3}$ showing the four pairs of warped sheets. (b) Particular sections of the Fermi surface of $\mathrm{NbSe}_{3} . \Gamma=(0,0,0), Y=(1 / 2,0,0), Z=(0,1 / 2,0), C=$ $(1 / 2,1 / 2,0), B=(0,0,1 / 2), D=(0,1 / 2,1 / 2), A=(1 / 2,0,1 / 2)$, and $E=(1 / 2,1 / 2,1 / 2)$ in units of the monoclinic reciprocal lattice vectors. briefly discuss the result that would have been observed if the system were an usual 3D material (Sec. VB), then develop symmetry rules for the $1 \mathrm{D}$ case (Sec. V C), and analyze the outcome separately for each column (Secs. V D and VE). We conclude that the experimental data imply that, in contrast to the DFT results, the coherent motion of the electrons within the $a-c$ plane is inhibited, endowing $\mathrm{NbSe}_{3}$ with a stronger 1D character than anticipated.

\section{A. Definitions}

ARPES is a probe where a high frequency electromagnetic wave suddenly removes an electron from a given electronic orbital. We probe the propagation (and subsequent recombination) of an excited photohole. The ARPES intensity is approximately given by (see, e.g., [54])

$$
I(\vec{A}, \vec{q}, \omega)=\left|M_{f i}(\vec{A})\right|^{2} \mathrm{~A}(\vec{q}, \omega) f(\omega),
$$

where $\omega$ and $q$ are, respectively, the energy and the in-plane momentum deposited by the photon, and $\vec{A}$ is the vector potential of the photon field. $\mathrm{A}(\vec{q}, \omega)$ is the one-particle spectral function, and $f$ is the Fermi factor [55]. $\left|M_{f i}(\vec{A})\right|^{2}$ is the matrix element we will focus on in this section. It depends on the shape of the initial and final states and the photon polarization.

The matrix elements are defined between one-body wave functions in the initial and final state:

$$
M_{f i}(\vec{A})=\left\langle\psi_{f}|\vec{A} \cdot \overrightarrow{\hat{P}}| \psi_{i}\right\rangle,
$$

where $\hat{P}$ is the momentum operator. Typically $\left|\psi_{f}\right\rangle$ will have the symmetry of a plane wave whose wave vector $\vec{k}_{f}$ defines, together with the incident photon wave vector, the scattering plane. In the following we denote it $\sigma_{l}$. The polarization of the incident light can thus be perpendicular to this plane ( $s$ polarization; $\vec{A} \perp \sigma_{l}$ ) or within the plane $\sigma_{l}$ ( $p$ polarization). Note that if we choose a gauge with zero scalar potential we can rewrite the interaction term as $\vec{E} \cdot \overrightarrow{\hat{R}}$, where $\hat{R}$ is the position operator. This makes the symmetries of (2) more transparent, 
especially if one rewrites the states as real space wave functions $\left|\psi_{i}\right\rangle \equiv \psi_{i}(\vec{r})$ and integrates over space to obtain $M_{f i}(\vec{A})$.

In the following we use a shorthand bra-ket notation $|e, o\rangle$ to indicate an even/odd state with respect to the plane $\sigma_{l}$. The final state $\left|\psi_{f}\right\rangle$ is always even, thus $|e\rangle$, because it can be expressed as an even parity plane-wave state on the detector's surface [52]. Therefore, we have two possibilities depending on the polarization. For $s$ polarization the initial state $\left|\psi_{i}\right\rangle$ must be odd with respect to the plane $\sigma_{l}$ to make the overall space integral $M_{i f} \sim\langle e|o| o\rangle \neq 0$. On the contrary, for $p$ polarization the initial state $\left|\psi_{i}\right\rangle$ must be even.

In most systems, electron hopping can be considered reasonably isotropic, and the electronic states are well represented by Wannier functions, which are coherent superpositions in the three directions of the local orbitals. $\left|\psi_{i}\right\rangle$ is a quantum coherent sum of these local entities, which we need to sum to get the $M_{i f}$. Actually, this summation allows us to pick a state with a well defined quasimomentum inside a crystal. We see that, in order to have a well defined symmetry, the plane $\sigma_{l}$ must coincide with a true symmetry plane $\Sigma$ of the 3D crystal lattice [52]. Then the bands observed in $s$ and $p$ polarization can be different. If this is not the case, then there is no symmetry protection and there will be in general a mixing of the two signals. In the $\mathrm{NbSe}_{3}$ crystal lattice, where the space group is $P 2_{1} / m$, there are two possible $\Sigma$ planes, both parallel to the $a c$ plane [see Fig. 1(a)], thus perpendicular to the $\sigma_{l}$ plane. We conclude that, if the system could be reasonably described as a three-dimensional material, the spectra should be essentially independent of light polarization.

\section{B. Rough selection rules}

Note that in addition to the symmetry selection rules above, the strength of interaction with light does depend on the shape of the orbitals which are at the root of the Wannier functions. From (2) we see that the amplitude is expected to be larger, the electric dipole moment $\varrho_{j}$ bigger, when the transition involves a $j$ orbital that is extended in the direction given by $\vec{E}$. The orbitals spread along the $c$ axis are likely to be led to larger visibility with the $s$-polarized light, while those which have a large cross section within the $\sigma_{l}$ plane will be more visible in the $p$-polarized light. From this we deduce that the $p_{y}$ component of Se occupancy (and a minor $d_{x y}$ component of $\mathrm{Nb}$ occupancy as well) will favor interaction with $s$-polarized light while all other components with $p$-polarized light.

Such "rough selection rules" are based on a single electron 3D description. Both tight binding and DFT result yield the atomic content for each electronic band near the Fermi level. It is therefore possible to predict their visibility according to this rough selection rule. There are two tests that we can run against our experimental data: one qualitative and one quantitative. Quantitatively, we notice that the $p_{y}$ component is about half of the total Se (for atoms on short bond) occupancy while the probability of finding one electron from the A1/3 band on Se atom is approximately $1 / 3$ (note that apical Se atoms have less $p_{y}$ content). Hence the ratio of intensities of $s$ vs $p$ polarization should be (at most) $\left([0.5 * 0.3(3)+0.1 * 0.6(6)]^{2} /\{1-[0.5 * 0.3(3)+\right.$ $\left.\left.0.1 * 0.6(6)]^{2}\right\}\right)=0.058\left(\right.$ and here we added an extra $10 \% d_{x y}$ component of $\mathrm{Nb}$ occupancy). We conclude that, if we were observing the same $3 D$ state in both polarizations, then such state would be visible above the noise level only in one polarization, in clear contrast with the result of our experiment. This prediction clearly contrasts with the experimentally observed ratio $\approx 0.5$. Actually, since the beamline throughput is larger for $p$ polarization, the intrinsic ratio is even closer to 1.0 (i.e., equal intensities for both polarizations).

Qualitatively, one observes a substantial drop in the intensity of the A3 band at binding energy $0.25 \mathrm{eV}$, but only in $s$ polarization. The orbital components do not exhibit any anomaly for the corresponding momenta. For $k_{c}, k_{a} \approx 0$ we see only a very weak dependence of orbital content on momentum and nearly identical orbital content for the A1 and A3 bands (in accordance with an intuitive two-leg ladder model proposed below). Actually, no sensible model for lattice hybridization could explain a sudden variation of A3 content without any visible change of A1 content.

Finally, one could point out that the different sensitivities of different atoms to light at a given energy should be taken into account. Indeed, in our case there is a larger photoionization cross section for the Se atomic sites, but at the same time the hybridization along the $b$ axis is much stronger than along the $c$ axis (note that for the moment, for the sake of argument we are considering 3D system, but it is an anisotropic 3D system) hence the elongation is larger along the former direction. It is safe to assume that these two secondary effects compensate each other.

\section{New (1D) selection rules}

The summation that defines $\left|\psi_{i}\right\rangle$ is done over a quantum coherent part of the electron liquid. Thus new possibilities for $\Sigma$ arise in a quasi-one-dimensional material. If the hopping between one-dimensional entities, which will be discussed below, is small compared to the inverse timescale of the ARPES process $t_{\perp} \ll t<\hbar \omega$, hopping in the direction perpendicular to the chains is essentially incoherent $[16,17,56]$. In this high-energy limit the electron liquid is then in a $1 \mathrm{D}$ regime. At low energy the gaps $\Delta_{1,2}$ that appear in the spectrum (see [57]) also prevent single particle hopping between chains. Thus, in both limits the electronic waves perpendicular to the $b$ axis are incoherent. In that case, the matrix element in (2) must be computed for a single entity only, and thus the symmetry is based on a wave function for a single entity. One could think naively that the entities to be considered are the chains themselves, in which case any plane that would contain the columns [see Fig. 1(a)] could be a good symmetry plane. However, one should not neglect the col.A(1)-col.A(2) hybridization, which manifests itself as a splitting of the $b 1 / b 3$ bands. The symmetry plane $\Sigma$ must accommodate these strong links, and a plane perpendicular to the $b c$ plane must be chosen. This plane coincides with the scattering plane $\sigma_{l}$. We thus find that in the $1 \mathrm{D}$ regime $\Sigma=\sigma_{l}$ and that the selection rules are strictly obeyed. Let us now examine the consequences for each column or group of columns.

\section{Column C}

The prisms out of which col.C is built are strongly distorted. This means that the high symmetry of an ideal $D_{3 h}$ 


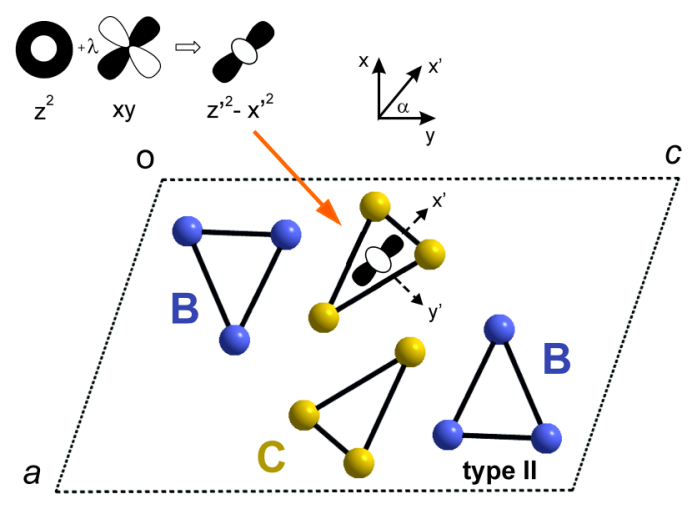

FIG. 16. Mixing of $\mathrm{Nb} d$ orbitals on column $\mathrm{C}$ yields composite orbitals visible in both polarizations.

chain, out of which the double prismatic columns are made, is lowered to $C_{2 h}$. The $d_{z^{\prime 2}}$ orbital, which is decoupled in a $D_{3 h}$ environment, for $C_{2 h}$ crystal field contains an admixture of $d_{x^{\prime 2}-y^{\prime 2}}$, because now $d_{z^{\prime 2}}$ and $d_{x^{\prime 2}-y^{\prime 2}}$ belong to the same, rank-1 symmetry class $a_{1}$. The two orbitals $\mathrm{Nb}(\mathrm{C})-d_{z^{\prime 2}}$ and $\mathrm{Nb}(\mathrm{C})-d_{x^{\prime 2}-y^{\prime 2}}$ remain split by the crystal field potential, but its effect can be effectively reduced owing to a relatively large value of the Hund coupling (one peculiarity of $\mathrm{Nb}$ [58]), which in a mean field treatment favors the symmetry-allowed orbital mixing.

So far we have used the primed system of coordinates, related to a local $\mathrm{Nb}$ atom, because the local crystal field is associated with a given $\mathrm{Nb}$ site, see Fig. 16. However, while the admixed orbital is the $d_{x^{\prime 2}-y^{\prime 2}}$ in the local system of coordinates, in the system of coordinates linked with the scattering plane $\sigma_{l}$ the spatial shape of the admixed $\mathrm{Nb} d$ orbital is very close to a $d_{x y}$ orbital. This is due to the tilting of the col.C prisms with respect to the $b c$ plane (the tilt angle is $\alpha=43^{\circ}$ ). This explains why C2 is visible in $s$ polarization. Actually, the amplitude of this $s$ polarization signal can be stronger than one could expect solely from a rather weak $\mathrm{Nb}-d_{x y}$ component. The $\mathrm{Nb}-\mathrm{Se}$ bond has mostly a covalent character, with nearly 50\% Se $p$ spectral weight. The admixed $d_{x y}$ has a larger overlap (than $d_{z^{2}}$ ) with the $p$-Se orbitals [including the $p$-Se(B)] and thus it plays a bigger role in $p$ - $d$ overlap integrals. These in turn determine the symmetry properties of the outer part of the "C2-molecular orbital," the $\psi_{i}^{\mathrm{C} 2}$ in our ARPES description. This expectation can be put on more solid ground. From our DFT calculation, we obtain that the $p$-Se part of the C2-molecular orbital is located on a short side of col.C prisms (as expected), the $\mathrm{Se}\left(\mathrm{C}^{<}\right)$atoms, and the Se-Se bond has mostly a $\pi$ character, turned away from $\mathrm{Nb}$ site, thus with larger Se $p_{y}$ component (in a system of coordinates of Fig. 16). Then indeed the $p$-Se part has stronger odd character vs $\sigma_{l}$. The local part of the initial wave function corresponding to band $\mathrm{C} 2$, this must be convoluted with Bloch wave along $b$ axis and antisymmetrized to get the full $\psi_{i}^{\mathrm{C} 2}(\vec{r})$, is a composite orbital:

$$
\begin{aligned}
\psi_{i}^{\mathrm{C} 2}(\vec{r})= & \sum a_{d_{z^{2}}} \chi_{d_{z^{2}}}\left(\vec{r}-R_{\mathrm{Nb}(\mathrm{C})}\right) \\
& +a_{d_{x y}} \chi_{d_{x y}}\left(\vec{r}-R_{\mathrm{Nb}(\mathrm{C})}\right)+a_{p_{y}} \chi_{p_{y}}\left(\vec{r}-R_{\mathrm{Se}\left(\mathrm{C}^{<}\right)}\right)+\cdots
\end{aligned}
$$

where $\chi_{j}\left(\vec{r}-\vec{R}_{0}\right)$ is an atomic orbital $j$ localized around a given site $\vec{R}_{0}$ and $a_{j}$ are coefficients of the expansion, the $\mathrm{Se}\left(\mathrm{C}^{<}\right)$indicates atoms on the shorter edge of the prism. Ellipsis accounts for further $p$-Se contributions present on all surrounding atoms. Assuming that there is no interaction between the outgoing photoelectron and other carriers (this is at the core of the sudden approximation), then the symmetry character of the composite orbital $\psi_{i}^{\mathrm{C} 2}(\vec{r})$ is a weighted sum of the symmetry characters of its atomic constituents. The $M_{f i}$ becomes a weighted sum of $a_{j}$ coefficients: $M_{f i}=\sum_{j} w_{j} a_{j}$, where only $a_{j}$ with an appropriate symmetry contribute. Since we have linked our system of coordinates to $\sigma_{l}$ it is straightforward to establish whether a given $\chi_{j}$ contributes or not for a given polarization. In the case of $\psi_{i}^{\mathrm{C} 2}(\vec{r})$ we have $\chi_{d_{z^{2}}} \equiv|e\rangle, \chi_{d_{x y}} \equiv|o\rangle$, and $\chi_{p_{y}} \equiv|o\rangle$ thus one expects: $M_{f i}^{\mathfrak{p}}=$ $\sum_{j} w_{d_{z^{2}}} a_{d_{z^{2}}}+\cdots$ and $M_{f i}^{\mathfrak{s}}=\sum_{j} w_{d_{x y}} a_{d_{x y}}+w_{p_{y}} a_{p_{y}}+\cdots$.

To evaluate $M_{f i}$ we need to know the weights $w_{j}=\varrho_{j} \bar{w}_{j}$, where $\rho_{j}$ is the already discussed geometric factor, which is certainly largest for a well delocalized orbital $p_{z}-\operatorname{Se}(\mathrm{C}) . \bar{w}_{j}$ is the bare photoionization cross section tabulated in Ref. [59]. For $\hbar \omega=100 \mathrm{eV}$ the $\mathrm{Nb} 4 d$ cross section is close to the Cooper minimum $[59,60]$, while the $\mathrm{Se} 4 p$ orbitals have a relatively large cross section (around eight times larger). Then, surprisingly, the Se $p$ part enters with larger weight. As a result the band $\mathrm{C} 2$ has a significant odd component with respect to $\sigma_{l}$ and thus it can be well visible in $s$ polarization.

An issue is why the even component of C2 is not well visible in $p$ polarization, since the new $1 \mathrm{D}$-selection rules and the DFT results imply that for $p$ polarization $M_{f i}$ is close to that of the odd part [61]. It should be noted that previous experiments, performed with different photon energies, revealed the doubly degenerated band $\mathrm{C} 2$ as the strongest, in accordance with the expectation from the DFT and the sudden electron approximation. Thus spectral weight is missing at the particular condition we probe. We need to search for a cause that depends on energy and on the fact that electrons are ordered. A possible explanation lies beyond the sudden electron approximation. For the chosen photon energy the wavelength of the excited photoelectrons matches well the distance between the nearest $\mathrm{Nb}(\mathrm{C})$ sites. This enhances photoelectrons backscattering, which reduces the ARPES signal. Mathematically, these processes would produce strong vertex corrections to $\bar{w}_{d_{z^{2}}}$, a coefficient that enters in $M_{i j}^{\mathfrak{p}}$. Only the $p$-polarization part of C2 is strongly affected, because this is the one that originates mostly from an on-site $\mathrm{Nb}(\mathrm{C})-d_{z^{2}}$.

\section{E. Column A}

Contrary to the wave function $\psi_{i}^{\mathrm{C} 2}$, which is localized around a given col.C, the "molecular orbitals" corresponding to well split bonding/antibonding bands A1 and A3, the $\psi_{i}^{\mathrm{A} 1}$ and $\psi_{i}^{\mathrm{A} 3}$, must be delocalized over an entire quartet col.A+col.B. The single column starting point cannot be taken. On the other hand, here the arrangement of most Se $p$ bonds is perpendicular to the $\sigma_{l}$ plane. Then, also in accordance with $\bar{w}_{\mathrm{Nb}}<\bar{w}_{\mathrm{Se}}$, it is more convenient to start with the Se $p$ part of the $\psi_{i}^{\mathrm{A} 1}$ and $\psi_{i}^{\mathrm{A} 3}$. In particular, we focus on Se $p-\left(\mathrm{A}^{<}\right)$, the orbitals around a short link between col.A-col.B, shown as a green link in Fig. 1. 
Focusing on Se $p$ - $\left(\mathrm{A}^{<}\right)$one can construct two states:

$$
\begin{aligned}
\psi_{i}^{\gamma+}(\vec{r})= & \sum_{R_{i}} a_{p_{y}}^{\gamma+} \chi_{p_{y}}\left(\vec{r}-R_{\operatorname{Se}\left(\mathrm{A}^{<}\right)}\right) \\
& +a_{p_{y}^{\prime}}^{\gamma+} \chi_{p_{y}}\left(\vec{r}-R_{\mathrm{Se}(\mathrm{A}, \mathrm{B})}\right) \\
& +a_{d_{z^{2}}^{\gamma+}}^{\gamma+} \chi_{z_{z^{2}}}\left(\vec{r}-R_{\mathrm{Nb}(\mathrm{A})}\right)+\cdots,
\end{aligned}
$$

where the second term is implied by the $\sigma$ character of the Se-Se bonds, as determined by the $p_{y}$ orientation, and

$$
\begin{aligned}
\psi_{i}^{\gamma-}(\vec{r})= & \sum_{R_{i}} a_{d_{z^{2}}}^{\gamma-} \chi_{d_{z^{2}}}\left(\vec{r}-R_{\mathrm{Nb}(\mathrm{A})}\right) \\
& +a_{p_{x, z}-}^{\gamma-} \chi_{p_{x, z}}\left(\vec{r}-R_{\mathrm{Se}\left(\mathrm{A}^{<}\right)}\right) \\
& +a_{p_{x, z}^{\prime}}^{\gamma-} \chi_{p_{x, z}}\left(\vec{r}-R_{\mathrm{Se}(\mathrm{A}, \mathrm{B})}\right)+\cdots,
\end{aligned}
$$

where we take a sum over all atomic orbitals within a double unit cell that constitute a given eigenstate in real space. By reasoning along the same lines like as for $\psi_{i}^{\mathrm{C} 2}$, taking $\chi_{d_{z^{2}}} \equiv|e\rangle, \chi_{p_{y}} \equiv|o\rangle$, and $\chi_{p_{x, z}} \equiv|e\rangle$, we deduce for the $\gamma+$ state: $M_{f i}^{\mathfrak{s} \gamma+}=\sum_{j} w_{p_{y}}\left(a_{p_{y}}^{\gamma+}+a_{p_{y}}^{\gamma+}\right)+\cdots, M_{f i}^{\mathfrak{p} \gamma+}=$ $\sum_{j} w_{d_{z^{2}}} a_{d_{z^{2}}}^{\gamma+}$. Since $\left(a_{p_{y}}^{\gamma+}+a_{p_{y}}^{\gamma+}\right)>a_{d_{z^{2}}}^{\gamma+}$ and $w_{p_{y}}>w_{d_{z^{2}}}$ we obtain $M_{f i}^{\mathfrak{s} \gamma+} \gg M_{f i}^{\mathfrak{p} \gamma+}$. For the $\gamma-$ state the selection rule is strictly obeyed: $M_{f i}^{\mathfrak{p} \gamma-}=\sum_{j} w_{d_{z^{2}}} a_{d_{z^{2}}}^{\gamma-}+w_{p_{x, z}}\left(a_{p_{x, z}}^{\gamma-}+a_{p_{x, z}}^{\gamma-}\right)+$ $\cdots, M_{f i}^{\mathfrak{s} \gamma-}=0$. One can also invert the reasoning to realize that we have one initial state that interacts strongly with $s$-polarized light, while the other only with $p$-polarized light. It is then quite reasonable to expect that a photohole created by $s$-polarized light $\left|\psi_{h}^{s}\right\rangle$ will have a large overlap with only one eigenstate, the $\gamma+$ shown in Fig. 17, that is $\left\langle\psi_{h}^{s} \mid \psi_{i}^{\gamma+}\right\rangle \approx 1$ and by analogy $\left\langle\psi_{h}^{p} \mid \psi_{i}^{\gamma-}\right\rangle \approx 1$. Then one observes a double dispersion of bands A1, A3 because the two different initial states are probed with the two different polarizations.

\section{THEORETICAL INTERPRETATION}

In the previous section we showed that ARPES selection rules can emerge due to the one-dimensional character of the material. We now need to check if the peculiar physics of $\mathrm{NbSe}_{3}$ can be captured in this situation. Obviously, the standard nesting scenario for the lower CDW transition, based on a warped 2D Fermi surface, must be abandoned. Note that such nesting scenario was already hardly compatible with results from $x$-ray diffuse scattering [62]. It has been proposed already long ago [27] that phase transitions in $\mathrm{NbSe}_{3}$ must be considered from the perspective of strong interactions, but so far experiments managed to avoid, albeit sometimes narrowly [21], to tackle this admittedly very complex theoretical issue. However, any nesting-based weak coupling analysis could hardly explain the observed doubling of the bands. Therefore, an interpretation of our results cannot avoid correlations (due to interactions) that are only partly taken into account in DFT calculations.

In order to see how interactions and low dimensionality could affect the DFT results, we first consider bands C2, which are degenerate and nearly commensurate, and hence act as a doublet of $1 \mathrm{D}$ chains, prone to a Peierls distortion. Indeed the $\mathrm{C} 2$ dispersion is clearly backfolded, an effect of the CDW1
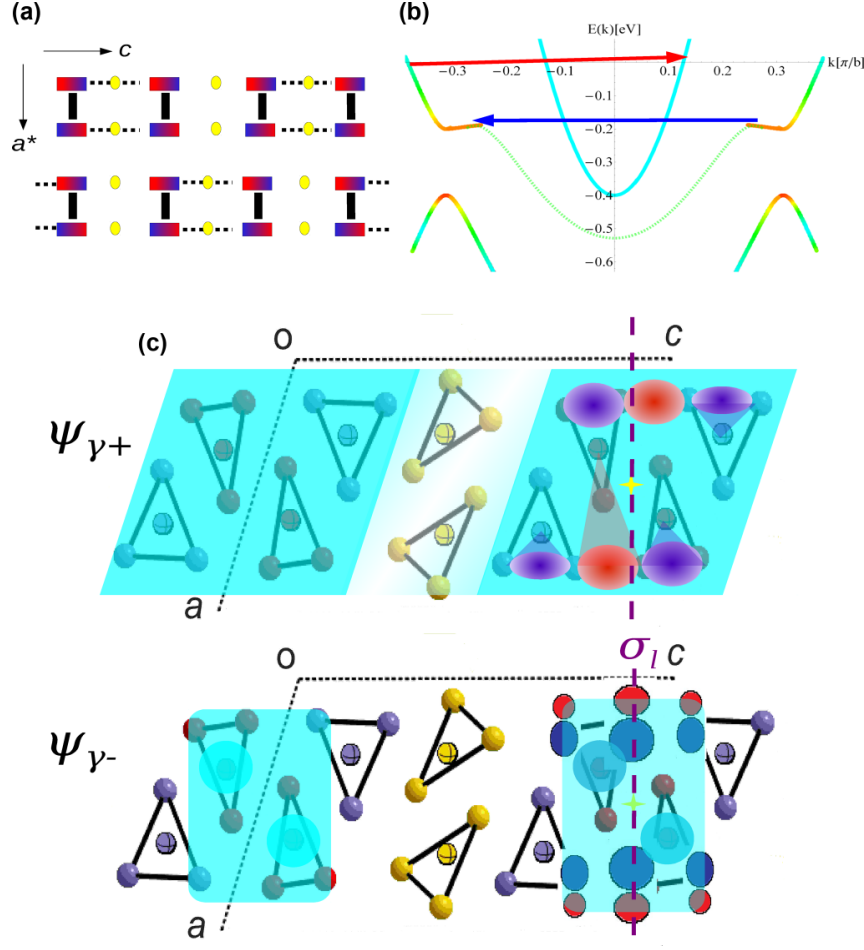

FIG. 17. (a) $a c$ plane cross section showing schematically the perpendicular dimerization and emergent self-organization. Yellow dots represent col.C where CDW1 takes place. Pairs of red-blue rectangles linked by a thick black line are doublets of $\mathrm{A}+\mathrm{B}$ columns. The perpendicular dimerization is indicated by the dashed lines. (b) Model band structure of rehybridized $\gamma+$ states showing the proximity of an interband $\mathrm{A} 3^{+} \rightarrow \mathrm{A} 1^{+}$backscattering (red arrows) to the CDW1 wave vector $\mathbf{q}_{1}$ (blue arrow). The color scale identifies the "hot spot" (c) (schematic) bonding $\gamma_{+}$and antibonding $\gamma_{-}$orbitals have a different spatial extension. Their amplitude is indicated by the strength of turquoise shading. Red and blue color indicates the phase of the involved $p$ orbitals. The orientation of a $p$ orbital determines the direction in which the resulting $\psi_{\gamma}$ orbital will be extended. $\gamma_{+}$ $\left(\gamma_{-}\right)$exhibits odd (even) character vs the $\sigma_{l}$ plane indicated by the dashed line.

with wave vector $\mathbf{q}_{1}$, for which a charge distortion is known to be located mostly on $\mathrm{C}$ columns $[23,25,27]$. The $1 \mathrm{D}$ character of this transition manifests itself in an anomalously large ratio $\left(\Delta_{1} / k_{B} T_{\mathrm{CDW} 1}\right)=6.7$ [57]. The CDW1 transition thus partitions the rest of the electronic liquid into $1 \mathrm{D}$ subsystems [63] located on quartets of $A+B$ columns, where the charge is predominantly on column A. Each pair of A columns acts as a two-leg ladder system, as shown in Fig. 17.

Such 1D substructure would be at the root of the observed polarization dependence. A complete calculation, taking into account the full ladder structure and its possible instabilities $[15,64-66]$, would be beyond the scope of the present paper and will be done elsewhere [67]. We offer here a plausible scenario, compatible with the experimental data. Let us denote by $\psi_{\mathrm{Ai}^{+}}(r)$ the wave function corresponding to a DFT band $\mathrm{Ai}^{+}$. Let us assume a spontaneous dimerization in the $a c$ plane, such transition is permitted since a system of mutually exclusive weak-strong bonds is in essence a 2D Ising model, so a discrete symmetry is broken upon transition. The transition is bringing closer two A-B blocks as in Fig. 17(a), 
then the $\psi_{\mathrm{Ai}}(r)$ orbital will deform, leading to bonding and antibonding combinations, denoted $\gamma^{ \pm}$in Fig. 17(c). These two combinations have very different interactions with the inner $\mathrm{C}$ column, which carries the periodic charge modulation associated with CDW1. For the bonding states $\psi_{\gamma^{+} 1,3}(r)$ the local coupling, due to the emerging overlap between $\psi_{\gamma^{+1,3}}(r)$ and the electronic orbitals on $\mathrm{C}$ columns, should thus enhance inter-band scattering with large momentum exchange [Fig. 17(b)]. The perpendicular deformation is energetically favorable because it allows to harvest electron-electron interaction energy available thanks to the closeness (in momentum space) of a col.A interband instability and col.C induced hot spot in the $\mathrm{A} 3^{+}$dispersion, indicated by red and blue arrows in Fig. 17(b). In the experiment, this manifests itself as a significant rehybridization with the gapped band C2, an effect clearly visible in Fig. 5(c) and already noticed but not directly addressed [21].

One consequence of this scenario is that the 1D CDW2 formation is accompanied by a dimerization along the $c$ axis. An organization in the $a c$ plane with a chessboard pattern as in Fig. 17(a) is a natural choice to minimize Coulomb repulsion. It naturally yields the CDW2 periodicity $q_{2}^{\perp}=\left(\frac{1}{2} a^{*}, \frac{1}{2} c^{*}\right)$, which is hard to justify in a standard nesting scenario. Moreover this ansatz directly solves several experimental riddles: the band doubling into $\mathrm{A} 1(3)^{+}$and $\mathrm{A} 1(3)^{-}$and their tiny splitting, the number of electrons that remain ungapped, and the presence of gapless carriers close to the $\Gamma$ point. The hopping between 1D subunits remains incoherent down to very low temperatures. In this case the effective symmetry plane for the $1 \mathrm{D}$ system consisting of a quartet of $\mathrm{A}+\mathrm{B}$ columns must: (i) be perpendicular to the $c$ axis, (ii) contain the $b$ axis, and (iii) cross the center of the quartet. The scattering plane $\sigma_{l}$ fulfils these conditions and thus the dipole selection rules emerge. The bonding states $\gamma^{+}$are by construction delocalized towards column C and involve $p$-Se $\sigma$ hybridization on the short link [green line in Fig. 1(b)]. These states are antisymmetric with respect to the $\sigma_{l}$ plane [see Fig. 17(c)] and hence are expected to appear in $s$ polarization, in agreement with the experiment. A $\pi$-bond component on the short bond, required to keep the total density close to the DFT solution, can be absorbed within the antibonding state $\gamma^{-}$. These states are symmetric (vs $\sigma_{l}$ ) and hence visible in $p$ polarization. Moreover, since they are mostly localized in the core of the quartet and interact weakly with CDW1, they are expected to be gapless. This is what we observe in our ARPES data.

\section{DISCUSSION AND CONCLUSION}

We wish to emphasize that results of all four preceding sections has to be taken altogether as a proof of the novel physics present in $\mathrm{NbSe}_{3}$. In experimental part we emphasized bands' backfolding in $s$-pol spectra (contrary to $p$-pol spectra) as evidence of partial CDW2, but actually a minimal, sufficient condition that could support our 1D scenario is a difference between the spectral functions measured in two polarizations. This has been obviously observed. The DFT $+U$ study shows a substantial agreement with experiment and in this allows us to narrow down the differences caused by further correlation effects. Furthermore, it allows for a precise estimate of ARPES intensities in two polarizations (although we use a three-step ARPES model, our confidence is strengthened by a recent study [68] which shows that within a one-step model, final state's effects cannot cause a substantial difference at a chosen photon energy) which questions the standard Fermiliquid scenario and favors the 1D scenario. The emerging 1D scenario indeed captures all details of our experimental data and is in fact in agreement with previous experimental findings. In particular, the new theory allows us to connect the rehybridization of bands $\mathrm{C} 2$ and $\mathrm{A} 3^{+}$, clearly visible in $s$-pol (but not in $p$-pol) and detected already before [21] (therein called "mysterious"), with the CDW gap formation, which makes the entire picture consistent.

In summary, we exploited ARPES with sub- $\mu$ m lateral resolution and polarization control to study the electronic states of $\mathrm{NbSe}_{3}$. We observed a strong dependence of the dispersion on the light polarization, incompatible with a simple anisotropic quasi-1D Fermi-liquid-like description of this material. Our results can be explained by a self-organization into orbitally distinct electronic systems. One set of electronic states is gapped as evidenced by backfolding of its electronic dispersion. The other exhibits one-dimensional character and remains metallic. The canonically accepted mechanism for the low-temperature CDW2 formation must be replaced by a new scenario where part of the carries couple more strongly with the preceding CDW1. Experimentally, our results demonstrate that pushing the lateral resolution of ARPES to submicron scale can lead to qualitative breakthroughs. This will be especially fruitful for studies of novel and strongly correlated materials, that were so far hindered by small crystal sizes and inhomogeneities. Theoretically, the new scenario for the CDW formation, based on self-differentiation leading to enhanced interaction with the preceding CDW, suggests a reinvestigation of other materials where similar sequences of phase transitions are present. These results open the way to new experiments searching for the emergence of hidden 1D dimensional states in strongly correlated materials.

\section{ACKNOWLEDGMENTS}

The work at Lausanne and Geneva is supported by the Swiss NSF. M.A.V. acknowledges financial support from Spanish MICIN Postdoctoral Mobility Program. S.P. acknowledges C'NANO Ile-de-France, DIM NanoK, for the support of the Nanospecs project. Work in Spain was supported by a MINECO (Grants FIS2015-64886-C5-4-P and CTQ2015-64579-C3-3-P), Generalitat de Catalunya (2017SGR1506 and 2017SGR1289), and XRQTC. E.C. acknowledges support from MINECO through the Severo Ochoa Centers of Excellence Program under Grant No. SEV-2015-0496. ICN2 is funded by the CERCA Program/Generalitat de Catalunya, and supported by the Severo Ochoa program from Spanish MINECO (Grant No. SEV2017-0706). P.A. acknowledges support from the Maria de Maeztu Units of Excellence Program under Grant MDM2017-0767. S.C. gratefully acknowledges the Becas Chile program (CONICYT PAI/INDUSTRIA 72090772) for a doctoral grant at the Universitat de Barcelona. Synchrotron SOLEIL is supported by the Centre National de la Recherche Scientifique (CNRS) and the Commissariat à l'Energie Atomique et aux energies Alternatives (CEA). 
[1] M. J. Lawler, K. Fujita, J. Lee, A. R. Schmidt, Y. Kohsaka, C. K. Kim, H. Eisaki, S. Uchida, J. C. Davis, J. P. Sethna, and E.-A. Kim, Intra-unit-cell electronic nematicity of the high$T_{c}$ copper-oxide pseudogap states, Nature (London) 466, 347 (2010).

[2] A. Mesaros, K. Fujita, H. Eisaki, S. Uchida, J. C. Davis, S. Sachdev, J. Zaanen, M. J. Lawler, and E.-A. Kim, Topological defects coupling smectic modulations to intra-unit-cell nematicity in cuprates, Science 333, 426 (2011).

[3] E. H. da Silva Neto, R. Comin, F. He, R. Sutarto, Y. Jiang, R. L. Greene, G. A. Sawatzky, and A. Damascelli, Charge ordering in the electron-doped superconductor $\mathrm{Nd}_{2-x} \mathrm{Ce}_{x} \mathrm{CuO}_{4}$, Science 347, 282 (2015).

[4] G. Ghiringhelli, M. Le Tacon, M. Minola, S. Blanco-Canosa, C. Mazzoli, N. B. Brookes, G. M. De Luca, A. Frano, D. G. Hawthorn, F. He, T. Loew, M. M. Sala, D. C. Peets, M. Salluzzo, E. Schierle, R. Sutarto, G. A. Sawatzky, E. Weschke, B. Keimer, and L. Braicovich, Long-range incommensurate charge fluctuations in (Y,Nd) $\mathrm{Ba}_{2} \mathrm{Cu}_{3} \mathrm{O}_{6+x}$, Science 337, 821 (2012).

[5] R. Comin, R. Sutarto, E. H. da Silva Neto, L. Chauviere, R. Liang, W. N. Hardy, D. A. Bonn, F. He, G. A. Sawatzky, and A. Damascelli, Broken translational and rotational symmetry via charge stripe order in underdoped $\mathrm{YBa}_{2} \mathrm{Cu}_{3} \mathrm{O}_{6+y}$, Science 347, 1335 (2015).

[6] E. Fradkin and S. A. Kivelson, High-temperature superconductivity: Ineluctable complexity, Nat. Phys. 8, 864 (2012)

[7] T.-M. Chuang, M. P. Allan, J. Lee, Y. Xie, N. Ni, S. L. Bud'ko, G. S. Boebinger, P. C. Canfield, and J. C. Davis, Nematic electronic structure in the "parent" state of the ironbased superconductor $\mathrm{Ca}\left(\mathrm{Fe}_{1-x} \mathrm{Co}_{x}\right)_{2} \mathrm{As}_{2}$, Science 327, 181 (2010).

[8] J. K. Glasbrenner, I. I. Mazin, H. O. Jeschke, P. J. Hirschfeld, R. M. Fernandes, and R. Valenti, Effect of magnetic frustration on nematicity and superconductivity in iron chalcogenides, Nat. Phys. 11, 953 (2015).

[9] S. A. Kivelson, E. Fradkin, and V. J. Emery, Electronic liquidcrystal phases of a doped Mott insulator, Nature (London) 393, 550 (1998).

[10] V. Oganesyan, S. A. Kivelson, and E. Fradkin, Quantum theory of a nematic Fermi fluid, Phys. Rev. B 64, 195109 (2001).

[11] Y. Wang, D. F. Agterberg, and A. Chubukov, Coexistence of Charge-Density-Wave and Pair-Density-Wave Orders in Underdoped Cuprates, Phys. Rev. Lett. 114, 197001 (2015).

[12] L. F. Tocchio, C. Gros, X.-F. Zhang, and S. Eggert, Phase Diagram of the Triangular Extended Hubbard Model, Phys. Rev. Lett. 113, 246405 (2014).

[13] T. Giamarchi, Quantum Physics in One Dimension (Oxford University Press, Oxford, 2004).

[14] V. V. Deshpande, M. Bockrath, L. I. Glazman, and A. Yacoby, Electron liquids and solids in one dimension, Nature 464, 209 (2010).

[15] P. Abbamonte, G. Blumberg, and A. Rusydi, A. Gozar, P. G. Evans, T. Siegrist, L. Venema, H. Eisaki, E. D. Isaacs, and G. A. Sawatzky, Crystallization of charge holes in the spin ladder of $\mathrm{Sr}_{14} \mathrm{Cu}_{24} \mathrm{O}_{41}$, Nature (London) 431, 1078 (2005).

[16] L. G. Caron and C. Bourbonnais, Renormalization of transverse hopping integral in quasi-1D conductors, Synth. Met. 27, A67 (1988).
[17] S. Biermann, A. Georges, A. Lichtenstein, and T. Giamarchi, Deconfinement Transition and Luttinger to Fermi Liquid Crossover in Quasi-One-Dimensional Systems, Phys. Rev. Lett. 87, 276405 (2001).

[18] D. J. Scalapino, First steps on the ladder, Nature (London) 377, 12 (1995).

[19] M. Grioni, S. Pons, and E. Frantzeskakis, Recent ARPES experiments on quasi-1D bulk materials and artificial structures, J. Phys.: Condens. Matter 21, 023201 (2009).

[20] Y. Oreg, E. Sela, and A. Stern, Fractional helical liquids in quantum wires, Phys. Rev. B 89, 115402 (2014).

[21] J. Schäfer, E. Rotenberg, S. D. Kevan, P. Blaha, R. Claessen, and R. E. Thorne, High-Temperature Symmetry Breaking in the Electronic Band Structure of the Quasi-One-Dimensional Solid $\mathrm{NbSe}_{3}$, Phys. Rev. Lett. 87, 196403 (2001).

[22] J. L. Hodeau, M. Marezio, C. Roucau, R. Ayroles, A. Meerschaut, J. Rouxel, and P. Monceau, Charge-density waves in $\mathrm{NbSe}_{3}$ at $145 \mathrm{~K}$ : Crystal structures, X-ray and electron diffraction studies, J. Phys. C 11, 4117 (1978).

[23] S. van Smaalen, J. L. de Boer, A. Meetsma, H. Graafsma, H.-S. Sheu, A. Darovskikh, P. Coppens, and F. Levy, Determination of the structural distortions corresponding to the $\mathbf{q}^{1}$ - and $\mathbf{q}^{2}$ type modulations in niobium triselenide $\mathrm{NbSe}_{3}$, Phys. Rev. B 45, 3103 (1992).

[24] S. Wada, R. Aoki, and O. Fujita, NMR study of the electronic state and Peierls transitions in $\mathrm{NbSe}_{3}$, J. Phys. F 14, 1515 (1984).

[25] C. Brun, Z.-Z. Wang, and P. Monceau, Scanning tunneling microscopy at the $\mathrm{NbSe}_{3}$ surface: Evidence for interaction between $\mathbf{q}_{\mathbf{1}}$ and $\mathbf{q}_{\mathbf{2}}$ charge density waves in the pinned regime, Phys. Rev. B 80, 045423 (2009).

[26] P. Monçeau, N. P. Ong, A. M. Portis, A. Meerschaut, and J. Rouxel, Electric Field Breakdown of Charge-Density-WaveInduced Anomalies in $\mathrm{NbSe}_{3}$, Phys. Rev. Lett. 37, 602 (1976).

[27] J.-P. Pouget, R. Moret, A. Meerchaud, L. Guemas, and J. Rouxel, X-ray observation of 1-d precursor effects in $\mathrm{NbSe}_{3}$ and new diffuse scattering features in $\mathrm{FeNb}_{3} \mathrm{Se}_{10}$, J. Phys. Colloques 44, C3-1729 (1983).

[28] A. Perucchi, L. Degiorgi, and R. E. Thorne, Optical investigation of the charge-density-wave phase transitions in $\mathrm{NbSe}_{3}$, Phys. Rev. B 69, 195114 (2004).

[29] N. Shima, Band structure of $\mathrm{NbSe}_{3}$, J. Phys. Soc. Jpn. 52, 578 (1983).

[30] E. Canadell, I. E. I. Rachidi, J. P. Pouget, P. Gressier, A. Meerschaut, J. Rouxel, D. Jung, M. Evain, and M. H. Whangbo, Comparison of the electronic structures of layered transition-metal trichalcogenides tantalum triselenide, tantalum trisulfide and niobium triselenide, Inorg. Chem. 29, 1401 (1990).

[31] J. Schäfer, M. Sing, R. Claessen, E. Rotenberg, X. J. Zhou, R. E. Thorne, and S. D. Kevan, Unusual Spectral Behavior of Charge-Density Waves with Imperfect Nesting in a Quasi-OneDimensional Metal, Phys. Rev. Lett. 91, 066401 (2003).

[32] C. W. Nicholson, C. Berthod, M. Puppin, H. Berger, M. Wolf, M. Hoesch, and C. Monney, Dimensional Crossover in a Charge Density Wave Material Probed by Angle-Resolved Photoemission Spectroscopy, Phys. Rev. Lett. 118, 206401 (2017).

[33] F. Lèvy and H. Berger, Single crystals of transition metal trichalcogenides, J. Cryst. Growth 61, 61 (1983). 
[34] J. Avila, I. Razado-Colambo, S. Lorcy, B. Lagarde, J.-L. Giorgetta, F. Polack, and M. C. Asensio, ANTARES, a scanning photoemission microscopy beamline at SOLEIL, J. Phys.: Conf. Ser. 425, 192023 (2013).

[35] J. Avila, A. Boury, B. Caja-Muñoz, C. Chen, S. Lorcy, and M. C. Asensio, Optimal focusing system of the fresnel zone plates at the synchrotron SOLEIL nanoARPES beamline, J. Phys.: Conf. Ser. 849, 012039 (2017).

[36] P. Zhang, P. Richard, T. Qian, Y.-M. Xu, X. Dai, and H. Ding, A precise method for visualizing dispersive features in image plots, Rev. Sci. Instrum. 82, 043712 (2011).

[37] J. E. Gayone, C. Kirkegaard, J. W. Wells, S. V. Hoffmann, Z. Li, and P. Hofmann, Determining the electron-phonon mass enhancement parameter $\lambda$ on metal surfaces, Appl. Phys. A 80, 943 (2005).

[38] A. A. Sinchenko and P. Monceau, Charge-density-wave gaps of $\mathrm{NbSe}_{3}$ measured by point-contact spectroscopy in different crystallographic orientations, Phys. Rev. B 67, 125117 (2003).

[39] K. Shimatake, Y. Toda, and S. Tanda, Selective optical probing of the charge-density-wave phases in $\mathrm{NbSe}_{3}$, Phys. Rev. B 75, 115120 (2007).

[40] F. H. L. Essler and A. M. Tsvelik, Finite Temperature Spectral Function of Mott Insulators and Charge Density Wave States, Phys. Rev. Lett. 90, 126401 (2003).

[41] P. Chudzinski and T. Giamarchi (unpublished).

[42] P. Hohenberg and W. Kohn, Inhomogeneous electron gas, Phys. Rev. 136, B864 (1964).

[43] W. Kohn and L. J. Sham, Self-consistent equations including exchange and correlation effects, Phys. Rev. 140, A1133 (1965).

[44] J. M. Soler, E. Artacho, J. D. Gale, A. García, J. Junquera, P. Ordejón, and D. Sánchez-Portal, The SIESTA method for ab initio order-N materials simulation, J. Phys.: Condens. Matter 14, 2745 (2002).

[45] E. Artacho, E. Anglada, O. Diéguez, J. D. Gale, A. García, J. Junquera, R. M. Martin, P. Ordejón, J. M. Pruneda, D. Sánchez-Portal, and J. M. Soler, The SIESTA method; developments and applicability, J. Phys.: Condens. Matter 20, 064208 (2008).

[46] J. P. Perdew, K. Burke, and M. Ernzerhof, Generalized Gradient Approximation Made Simple, Phys. Rev. Lett. 77, 3865 (1996).

[47] N. Troullier and J. L. Martins, Efficient pseudopotentials for plane-wave calculations, Phys. Rev. B 43, 1993 (1991).

[48] L. Kleinman and D. M. Bylander, Efficacious Form for Model Pseudopotentials, Phys. Rev. Lett. 48, 1425 (1982).

[49] E. Artacho, D. Sánchez-Portal, P. Ordejón, A. García, and J. M. Soler, Linear-scaling ab-initio calculations for large and complex systems, Phys. Status Solidi B 215, 809 (1999).
[50] S. L. Dudarev, G. A. Botton, S. Y. Savrasov, C. J. Humphreys, and A. P. Sutton, Electron-energy-loss spectra and the structural stability of nickel oxide: An LSDA $+U$ study, Phys. Rev. B 57, 1505 (1998).

[51] H. J. Monkhorst and J. D. Pack, Special points for Brillouinzone integrations, Phys. Rev. B 13, 5188 (1976).

[52] J. Hermanson, Final-state symmetry and polarization effects in angle-resolved photoemission spectroscopy, Solid State Commun. 22, 9 (1977).

[53] J. Mesot, M. Randeria, M. R. Norman, A. Kaminski, H. M. Fretwell, J. C. Campuzano, H. Ding, T. Takeuchi, T. Sato, T. Yokoya, T. Takahashi, I. Chong, T. Terashima, M. Takano, T. Mochiku, and K. Kadowaki, Determination of the Fermi surface in high- $T_{c}$ superconductors by angle-resolved photoemission spectroscopy, Phys. Rev. B 63, 224516 (2001).

[54] A. Damascelli, Z. Hussain, and Z.-X. Shen, Angle-resolved photoemission studies of the cuprate superconductors, Rev. Mod. Phys. 75, 473 (2003).

[55] G. D. Mahan, Many-Particle Physics, 3rd ed., Physics of Solids and Liquids (Kluwer Academic/Plenum, New York, 2000).

[56] M. Raczkowski, F. F. Assaad, and L. Pollet, Spin and charge dynamics of a quasi-one-dimensional antiferromagnetic metal, Phys. Rev. B 91, 045137 (2015).

[57] A. Fournel, J. P. Sorbier, M. Konczykowski, and P. Monceau, Measurement of the Charge-Density-Wave Gap of $\mathrm{NbSe}_{3}$ from Tunnel-Junction Spectra, Phys. Rev. Lett. 57, 2199 (1986).

[58] E. Şaşığlu, C. Friedrich, and S. Blügel, Effective Coulomb interaction in transition metals from constrained random-phase approximation, Phys. Rev. B 83, 121101 (2011).

[59] J. J. Yeh and I. Lindau, Atomic subshell photoionization cross sections and asymmetry parameters: $1<z<103$, Atom. Data Nucl. Data Tables 32, 1 (1985).

[60] J. W. Cooper, Photoionization from outer atomic subshells. A model study, Phys. Rev. 128, 681 (1962).

[61] A rough estimate gives 60(s-pol) : 40( $p$-pol $)$.

[62] S. Rouzière, S. Ravy, J. P. Pouget, and R. E. Thorne, X-ray investigation of the critical $\mathbf{q}_{2}$-charge density wave fluctuations of $\mathrm{NbSe}_{3}$, Solid State Commun. 97, 1073 (1996).

[63] The electrons are incoherent in the $a c$ plane at high energies due to the hopping anisotropy. See, e.g., Fig. 13.

[64] E. Dagotto, J. Riera, and D. Scalapino, Superconductivity in ladders and coupled planes, Phys. Rev. B 45, 5744 (1992).

[65] H. Tsunetsugu, M. Troyer, and T. M. Rice, Pairing and excitation spectrum in doped $t$-J ladders, Phys. Rev. B 49, 16078 (1994).

[66] H. J. Schulz, Phases of two coupled Luttinger liquids, Phys. Rev. B 53, R2959 (1996).

[67] P. Chudzinski and T. Giamarchi (unpublished).

[68] P. Chudzinski, Why it is so hard to detect Luttinger liquids in angle resolved photo-emission spectroscopy? J. Phys.: Condens. Matter 31, 105601 (2019). 